\title{
Comparing Voting by Committees According to their Manipulability*
}

\author{
R. Pablo Arribillaga ${ }^{\dagger} \quad$ Jordi Massó
}

September 3, 2016

\begin{abstract}
We consider the class of voting by committees to be used by a society to collectively choose a subset from a given set of objects. We offer a simple criterion to compare two voting by committees without dummy agents according to their manipulability. This criterion is based on the set-inclusion relationships between the two corresponding pairs of sets of objects, those at which each agent is decisive and those at which each agent is vetoer. We show that the binary relation "to be as manipulable as" endows the set of equivalence classes of anonymous voting by committees (i.e., voting by quotas) with a complete upper semilattice structure, whose supremum is the equivalence class containing all voting by quotas with the property that the quota of each object is strictly larger than one and strictly lower than the number of agents. Finally, we extend the comparability criterion to the full class of all voting by committees.
\end{abstract}

Keywords: Strategy-proofness, Separable Preferences, Voting by Committees. JEL classification: C78, D78.

${ }^{*}$ We are grateful to Dolors Berga and Bernardo Moreno, for very useful comments at an initial stage of this research, and to two referees of the journal, for their helpful and constructive comments. Arribillaga acknowledges financial support received from the Universidad Nacional de San Luis, through Grant 319502, and from the Consejo Nacional de Investigaciones Científicas y Técnicas (CONICET), through Grant PIP 112-200801-00655. Massó acknowledges financial support received from the Spanish Ministry of Economy and Competitiveness, through the Severo Ochoa Programme for Centres of Excellence in R\&D (SEV-20150563) and Grant ECO2014-53051-P, and from the Generalitat de Catalunya, through Grant SGR2014-515. The paper was partly written while Massó was visiting the Department of Economics at Stanford University; he wishes to acknowledge the hospitality of its members as well as financial support received from the Spanish Ministerio de Educación, Cultura y Deporte, through Project PR2015-00408.

†Instituto de Matemática Aplicada San Luis, Universidad Nacional de San Luis and CONICET. Ejército de los Andes 950, 5700 San Luis, Argentina. E-mail: rarribi@unsl.edu.ar

${ }^{\ddagger}$ Universitat Autònoma de Barcelona and Barcelona GSE. Departament d’Economía i Història Econòmica. Edifici B, UAB. 08193, Bellaterra (Barcelona), Spain. E-mail: jordi.masso@uab.es 


\section{Introduction}

In this paper we compare voting by committees according to their manipulability when they operate on the full domain of preference profiles, and hence they are manipulable. To do so, we apply a criterion introduced in Arribillaga and Massó (2016). Specifically, we consider a set of agents who have to collectively choose a subset from a given set of objects $K$. There are many social choice problems where the set of social alternatives is the family of all subsets of a given set. For instance, when the set of agents is the tenured members of a department and the set of objects is the set of junior candidates under consideration to become new assistant professors. Or a scientific society whose current fellows have to elect new fellows from a given list of candidates. Voting by committees (a subclass of social choice functions mapping preferences profiles into subsets of $K$ ) have been proposed to solve this class of problems. Following Barberà, Sonnenschein, and Zhou (1991) voting by committees are defined by specifying for each object $x \in K$ a monotonic family of winning coalitions $\mathcal{W}_{x}$ (a committee). Then the choice of the subset of objects made by a voting by committees at a preference profile is done object-by-object as follows. Fix a voting by committees $\mathcal{W}=\left(\mathcal{W}_{x}\right)_{x \in K}$ and a preference profile, and consider object $x$. Then, $x$ belongs to the chosen set (the one selected by $\mathcal{W}$ at the preference profile) if and only if the set of agents whose best subset of objects contains $x$ belongs to the committee $\mathcal{W}_{x}$. Hence, voting by committees can be seen as a family of extended majority voting (one for each object $x$ ) where the two alternatives at stake are whether or not $x$ belongs to the collectively chosen subset of objects.

A social choice function is strategy-proof if it always in the best interest of agents to reveal their preferences truthfully and it respects voter sovereignty if all subsets of objects can be chosen, for some revealed profile of agents' preferences. An agent's preferences are separable on the family of all subsets of objects $2^{K}$ if they are guided by the partition separating the set of objects into the set of good objects (as singleton sets, objects that are better than the empty set) and bad objects (as singleton sets, objects that are worse than the empty set). Adding a good object to any set leads to a better set, while adding a bad object leads to a worse set. Note that all additively representable preferences are separable. One of the main reasons why voting by committees are attractive is that they constitute the class of all strategy-proof social choice functions respecting voter sovereignty on the domain of separable preference profiles. So, voting by committees are attractive because they induce good strategic incentives to agents whenever they have separable preferences. But in addition they are appealing because they are simple. ${ }^{1}$

\footnotetext{
${ }^{1}$ See Barberà, Sonnenschein, and Zhou (1991) for a description of this class of problems and this axiomatic characterization of voting by committees.
} 
Agent $i$ is dummy at object $x$ in the committee $\mathcal{W}_{x}$ if $i$ does not belong to any minimal winning coalition of $\mathcal{W}_{x}$; that is, $i$ 's opinion about object $x$ is not used at all in the decision of whether or not $x$ belongs to the chosen subset. An especially interesting subclass of voting by committees are those without dummy agents. And among the class of voting by committees without dummy agents the subclass of voting by quotas is particularly appealing. A voting by committees is voting by quotas if the set of winning coalitions for each object $x$ are the sets of agents with equal or larger cardinality than a given strictly positive integer $q_{x}$, the quota of $x$. Hence, in any voting by quotas all agents play a symmetric role when determining whether or not objects belong to the chosen subset. Using the main characterization result in Barberà, Sonnenschein, and Zhou (1991) it is easy to see that the class of all anonymous and strategy-proof social choice functions satisfying voter sovereignty on the domain of separable preference profiles coincides with all voting by quotas.

Voting by committees are simple for two reasons. First, they are tops-only because they only depend on the profile of top subsets of objects, one for each agent. Second, they are object-by-object decomposable, and this is precisely the reason why they are strategy-proof on the domain of separable preference profiles: agent $i$, when considering whether or not to give support to object $x$, does not need to know the other set of elected objects because $i$ wants to support $x$ if and only if $x$ is a good object as a singleton set (i.e., $x$ belongs to $i$ 's best subset of objects) since its addition improves, by separability, any subset of objects.

However, in many applications the set of conceivable preferences of agents may be larger than the set of separable preferences: when adding an object to a set, some considerations regarding complementarities or substitutabilities among the added object and the objects already in the set may become relevant, yet they are not admissible if preferences are separable. For instance, although a voter for new assistant professors in the department considers that candidates $x$ and $y$ are the best and the second best candidates, both working in a similar research area, the voter may consider that, for the sake of diversity, the subset $\{x, z\}$ is better than the subset $\{x, y, z\}$, where $z$ is a third good candidate who works in a very different area from the one that $x$ and $y$ work, contravening separability. For this and similar cases we know that non-trivial voting by committees become manipulable, once non separable preferences are admitted in the domain where they operate. But since separable preferences may be conceivable too, voting by committees have still to be used to ensure that the social choice procedure remains strategy-proof on the subdomain of separable preference profiles. But the large mechanism design literature characterizing strategy-proof social choice functions on restricted domains of preferences has mainly neglected the potential interest to compare two social choice functions (operating on the full domain of preferences) according to their manipulability. And this is specially relevant if the designer has some doubts on whether agents' preferences are indeed restricted. 
In Arribillaga and Massó (2016) we have already argued that the manipulability of a social choice function does not indicate the degree of its lack of strategy-proofness. There may be only one instance at which the social choice function is manipulable or there may be many such instances. The mechanism design literature contains alternative measures (or lower and upper bounds) of the degree of manipulability to be applied to a given social choice function - see for instance Peleg (1979), Nitzan (1985), Kelly (1993), Smith (1999), Ehlers, Peters, and Storcken (2004), Maus, Peters, and Storcken (2007a, 2007b, 2007c, 2007d), Campbell and Kelly (2009) and Carroll (2013) — or direct criteria (in the form of partial orders) to compare pairs of social choice functions according to their manipulability - see for instance Pathak and Sönmez (2013), Chen, Egesdal, Pycia, and Yenmez (2016) and Arribillaga and Massó (2016).

As in Arribillaga and Massó (2016) our criterion to compare two social choice functions takes the point of view of individual agents. We say that an agent is able to manipulate a social choice function at a preference (the true one) if there exist preference relations, one for each of the other agents, and another preference for the agent (the strategic one) such that if submitted, the social choice function selects a strictly better alternative according to the agent's true preference. Consider two voting by committees, $\mathcal{W}$ and $\mathcal{V}$, operating on the universal domain of preference profiles. Assume that for each agent the set of preferences under which the agent is able to manipulate $\mathcal{W}$ is contained in the set of preferences under which the agent is able to manipulate $\mathcal{V}$. Then, from the point of view of all agents, $\mathcal{V}$ is more manipulable than $\mathcal{W}$. Hence, we think that $\mathcal{W}$ is unambiguously a better voting by committees than $\mathcal{V}$ according to the strategic incentives induced to agents. ${ }^{2}$ Often, it may be reasonable to think that agents' preferences are separable, but if the designer foresees that agents also may have non-separable preferences, then $\mathcal{W}$ may be a better choice than $\mathcal{V}$ if strategic incentives are relevant and important to the designer.

Before presenting our general result in Theorem 2, we focus on voting by committees without dummy agents. In Theorem 1 we provide a simple necessary and sufficient condition for the comparability of two voting by committees without dummy agents in terms of their

\footnotetext{
${ }^{2}$ Pathak and Sönmez (2013) proposed two comparability criteria based on the inclusion of sets of preference profiles and use them to compare two different matching mechanisms (in school choice problems) according to their manipulability. In contrast, our notion is based on the inclusion of sets of preference relations at which an agent is able to manipulate. In applications, preference profiles are not common knowledge while each agent knows his preference relation. A more manipulable social choice function requires that each agent has to worry about his potential capacity to manipulate in a larger set. In a remark at the end of the paper we present the two Pathak and Sönmez (2013)'s criteria and relate them to our comparability criterion. Chen, Egesdal, Pycia, and Yenmez (2016) proposed an agent-by-agent comparability criterion to compare stable matching mechanisms based on the inclusion of sets of alternatives that represent an improvement that an agent, at a preference profile, can obtain by misreporting.
} 
manipulability. This condition reflects the power of agents to influence the choice of the objects in the two voting by committees. Two notions are relevant to describe this power. Fix a voting by committees $\mathcal{W}=\left(\mathcal{W}_{x}\right)_{x \in K}$, an agent $i$, and an object $x$. We say that agent $i$ is decisive at $x$ if the singleton set $\{i\}$ belongs to $\mathcal{W}_{x}$; that is, $\{i\}$ is a winning coalition of $x$ and hence, $i$ can impose object $x$ in the final chosen subset by voting for $x$ (i.e., by declaring that $x$ belongs to $i$ 's top subset of objects). We say that agent $i$ is vetoer at $x$ if $i$ belongs to all winning coalitions of $\mathcal{W}_{x}$; that is, $i$ can make sure that $x$ is not in the final chosen subset by not voting for $x$ (i.e., by declaring that $x$ does not belong to $i$ 's top subset of objects). Then, the voting by committees without dummy agents $\mathcal{V}$ is more manipulable than the voting by committees without dummy agents $\mathcal{W}$ if and only if, for each agent, the decisive and vetoer sets of objects at $\mathcal{V}$ are contained respectively in the decisive and vetoer sets of objects at $\mathcal{W}$. Given the binary relation "to be as manipulable as" defined on the family of voting by committees we construct the set of equivalence classes of voting by committees, relative to this binary relation, by grouping together the voting by committees that are equally manipulable to each other, and extend this relation to the quotient set of equivalence classes in the natural way. Then we show that, when the number of agents $n$ is greater than or equal to three, the binary relation "to be at least as manipulable as" defined on the family of equivalence classes of voting by quotas (i.e., anonymous voting by committees) is a complete upper semilattice, whose maximal element is the equivalence class containing all voting by quotas where all quotas are strictly larger than 1 and strictly smaller than $n$. In the other hand, the equivalence classes of voting by quotas that are not more manipulable than any other equivalence class of voting by quotas are those where all objects have either quota 1 or quota $n$. We also identify, in Proposition 2, among all voting by committees without dummies (a larger set than the set of voting by quotas) those that are less manipulable. They can be characterized by two properties. First, the set of objects at which each agent is decisive contains the set of objects at which all agents are not vetoers. Second, the set of objects at which each agent is not a vetoer is contained in the set of objects at which some agent is decisive. In Theorem 2 we give the necessary and sufficient condition for the comparability according to their manipulability of two voting by committees, potentially with dummy agents. The condition is more involved than the one used to compare voting by committees without dummy agents and it incorporates, in addition to the inclusion of sets of objects at which each agent is decisive and vetoer, the inclusion of sets of objects at which each agent is a dummy. However, the inclusions of the three sets of objects are not necessary, but we show that they may not hold only in two very special circumstances, that we fully identify.

The paper is organized as follows. Section 2 contains preliminary notation and definitions. Separable preferences and voting by committees are defined in Section 3. Section 4 presents 
preliminary results. Section 5 compares voting by committees without dummy agents and Theorem 2 in Section 6 provides the complete criterion to compare any pair of voting by committees. Section 7 relates our comparability criterion with the two criteria proposed by Pathak and Sönmez (2013).

\section{Preliminaries}

Agents are the elements of a finite set $N=\{1, \ldots, n\}$. The set of objects is a finite set $K$. Generic agents will be denoted by $i$ and $j$ and generic objects by $x$ and $y$. Subsets of agents will be represented by $S$ and $T$ and subset of objects by $A$ and $B$. The set of agents $N$ has to choose a subset of $K$. Hence, the set of alternatives is the family $2^{K}$ of all subsets of objects. Given $S \subseteq N$ and $A \in 2^{K}$ we denote by $|S|$ and $|A|$ their cardinalities and by $\bar{S}$ and $\bar{A}$ their complementary sets. We assume that $|N|=n \geq 2$ and $|K| \geq 2$.

The (strict) preference of each agent $i \in N$ is a linear order $P_{i}$ on the set of alternatives; namely, $P_{i}$ is a complete, antisymmetric and transitive binary relation on $2^{K}$. As usual, let $R_{i}$ denote the weak preference relation induced by $P_{i}$; namely, for all $A, B \in 2^{K}, A R_{i} B$ if and only if either $A P_{i} B$ or $A=B$. The top alternative according to $P_{i}$ is the most preferred alternative and we denote it by $t\left(P_{i}\right)$; i.e., $t\left(P_{i}\right) P_{i} A$ for all $A \in 2^{K} \backslash\left\{t\left(P_{i}\right)\right\}$. Let $\mathcal{P}$ be the set of all preferences. A preference profile $P=\left(P_{1}, \ldots, P_{n}\right) \in \mathcal{P}^{n}$ is an $n$-tuple of preferences, one for each agent. To emphasize the role of agent $i$ a preference profile $P$ will be represented by $\left(P_{i}, P_{-i}\right)$.

A Cartesian product subset $\widehat{\mathcal{P}}^{n} \subseteq \mathcal{P}^{n}$ of preference profiles (or the set $\widehat{\mathcal{P}}$ itself) will be called a domain. A social choice function is a function $f: \widehat{\mathcal{P}}^{n} \rightarrow 2^{K}$ selecting, for each preference profile $P$ in the domain $\widehat{\mathcal{P}}^{n}$, a subset of objects $f(P) \in 2^{K}$.

A social choice function $f: \widehat{\mathcal{P}}^{n} \rightarrow 2^{K}$ satisfies voter sovereignty if for all $A \in 2^{K}$ there exists a profile $P \in \widehat{\mathcal{P}}^{n}$ such that $f(P)=A$; namely, $f$ is onto.

A social choice function $f: \widehat{\mathcal{P}}^{n} \rightarrow 2^{K}$ is tops-only if for all $P, P^{\prime} \in \widehat{\mathcal{P}}^{n}$ such that $t\left(P_{i}\right)=t\left(P_{i}^{\prime}\right)$ for all $i \in N, f(P)=f\left(P^{\prime}\right)$.

A social choice function requires that each agent reports a preference on a domain $\widehat{\mathcal{P}}$. A social choice function is strategy-proof on $\widehat{\mathcal{P}}^{n}$ if it is always in the best interest of agents to reveal their preferences truthfully. Formally, a social choice function $f: \widehat{\mathcal{P}}^{n} \rightarrow 2^{K}$ is strategy-proof if for all $P \in \widehat{\mathcal{P}}^{n}$, all $i \in N$, and all $P_{i}^{\prime} \in \widehat{\mathcal{P}}$,

$$
f\left(P_{i}, P_{-i}\right) R_{i} f\left(P_{i}^{\prime}, P_{-i}\right) .
$$

That is, a social choice function $f: \widehat{\mathcal{P}}^{n} \rightarrow 2^{K}$ is strategy-proof (on the domain $\widehat{\mathcal{P}}^{n}$ ) if, for each preference profile $P \in \widehat{\mathcal{P}}^{n}$ and each agent, truth-telling is a weakly dominant strategy in the normal form game induced by $f$ at $P$. We will say that a social choice function $f: \widehat{\mathcal{P}}^{n} \rightarrow 2^{K}$ 
is not manipulable by $i \in N$ at $P_{i} \in \widehat{\mathcal{P}}$ if $(1)$ holds for all $\left(P_{i}^{\prime}, P_{-i}\right) \in \widehat{\mathcal{P}}^{n}$. To compare social choice functions according to their manipulability, our reference set of preferences will be the full set $\mathcal{P}$.

The set of manipulable preferences of $i \in N$ at $f: \mathcal{P}^{n} \rightarrow 2^{K}$ is given by

$$
\mathcal{M}_{i}^{f}=\left\{P_{i} \in \mathcal{P} \mid f\left(P_{i}^{\prime}, P_{-i}\right) P_{i} f\left(P_{i}, P_{-i}\right) \text { for some }\left(P_{i}^{\prime}, P_{-i}\right) \in \mathcal{P}^{n}\right\}
$$

Obviously, a social choice function $f: \mathcal{P}^{n} \rightarrow 2^{K}$ is strategy-proof if and only if $\mathcal{M}_{i}^{f}=\{\emptyset\}$ for all $i \in N$. We say that $f: \mathcal{P}^{n} \rightarrow 2^{K}$ is more manipulable than $g: \mathcal{P}^{n} \rightarrow 2^{K}$ for $i \in N$ if $\mathcal{M}_{i}^{g} \subsetneq \mathcal{M}_{i}^{f}$.

Now, we introduce our criteria to compare social choice functions according to their manipulability.

Definition 1 A social choice function $f: \mathcal{P}^{n} \rightarrow 2^{K}$ is at least as manipulable as social choice function $g: \mathcal{P}^{n} \rightarrow 2^{K}$ if $\mathcal{M}_{i}^{g} \subseteq \mathcal{M}_{i}^{f}$ for all $i \in N$.

Definition 2 A social choice function $f: \mathcal{P}^{n} \rightarrow 2^{K}$ is equally manipulable as social choice function $g: \mathcal{P}^{n} \rightarrow 2^{K}$ if $f$ is at least as manipulable as $g$ and vice versa; i.e., $\mathcal{M}_{i}^{g}=\mathcal{M}_{i}^{f}$ for all $i \in N$.

Definition 3 A social choice function $f: \mathcal{P}^{n} \rightarrow 2^{K}$ is more manipulable than social choice function $g: \mathcal{P}^{n} \rightarrow 2^{K}$ if $f$ is at least as but not equally manipulable as social choice function g; i.e., $\mathcal{M}_{i}^{g} \subseteq \mathcal{M}_{i}^{f}$ for all $i \in N$ and there exists $j \in N$ such that $\mathcal{M}_{j}^{g} \varsubsetneqq \mathcal{M}_{j}^{f}$.

Given two social choice functions $f: \mathcal{P}^{n} \rightarrow 2^{K}$ and $g: \mathcal{P}^{n} \rightarrow 2^{K}$ we write (i) $f \succsim g$ to denote that $f$ is at least as manipulable as $g$, (ii) $f \approx g$ to denote that $f$ is equally manipulable as $g$, and (iii) $f \succ g$ to denote that $f$ is more manipulable than $g$. Obviously, there are many pairs of social choice functions that cannot be compared according to their manipulability.

\section{Separable Preferences and Voting by Committees}

Barberà, Sonnenschein, and Zhou (1991) characterizes, on the restricted domain of separable preferences, the family of all strategy-proof social choice functions satisfying voter sovereignty as the class of voting by committees. A preference $P_{i}$ is separable if the division between good objects ( $x$ is a good object if $\left.\{x\} P_{i}\{\emptyset\}\right)$ and bad objects ( $x$ is a bad object if $\{\emptyset\} P_{i}\{x\}$ ) guides the ordering of (some) subsets in the sense that adding a good object to any set leads to a better set, while adding a bad object to any leads to a worse set. Formally, 
Definition 4 A preference $P_{i} \in \mathcal{P}$ on $2^{K}$ is separable if for all $A \in 2^{K}$ and $x \notin A$,

$$
A \cup\{x\} P_{i} A \text { if and only if }\{x\} P_{i}\{\emptyset\} \text {. }
$$

Let $\mathcal{S}$ be the set of all separable preferences on $2^{K}$. Observe that for any separable preference its top is the subset of good objects. That is, for any separable preference $P_{i} \in \mathcal{S}$,

$$
t\left(P_{i}\right)=\left\{x \in K \mid\{x\} P_{i}\{\emptyset\}\right\}
$$

The following remark characterizes separable preferences. It follows from transitivity of the preference and it says that if we modify any given set of objects $A$ by removing good objects and adding bad objects, the new set is less preferred.

Remark $1 A$ preference $P_{i}$ is separable if and only if for all $A \in 2^{K}, T_{1} \subset t\left(P_{i}\right) \cap A$ and $T_{2} \subset \overline{t\left(P_{i}\right)} \cap \bar{A}$,

$$
A R_{i}\left(A \backslash T_{1}\right) \cup T_{2}
$$

We now define the class of social choice functions known as voting by committees. Let $x \in K$ be an object. A committee $\mathcal{W}_{x}$ for $x$ is a non-empty set of non-empty coalitions (subsets) of $N$, which satisfies the following monotonicity condition:

$$
\text { if } M \in \mathcal{W}_{x} \text { and } M \subset M^{\prime} \text {, then } M^{\prime} \in \mathcal{W}_{x} \text {. }
$$

A social choice function $f: \mathcal{P}^{n} \rightarrow 2^{K}$ is a voting by committees if for each $x \in K$ there exists a committee $\mathcal{W}_{x}$ such that for all $P \in \mathcal{P}^{n}$,

$$
x \in f(P) \text { if and only if }\left\{i \in N \mid x \in t\left(P_{i}\right)\right\} \in \mathcal{W}_{x}
$$

Observe that voting by committees are very simple. They are tops-only and the selected subset of objects at each preference profile is obtained in a decomposable way, object-byobject. Barberà, Sonnenschein, and Zhou (1991) characterizes this class when they operate on the restricted domain of separable preferences as follows.

Proposition 1 (Barberà, Sonnenschein, and Zhou, 1991) A social choice function $f: \mathcal{S}^{n} \rightarrow$ $2^{K}$ is strategy-proof and satisfies voter sovereignty if and only if it is voting by committees.

\section{Preliminary Results}

Let $\mathcal{W}_{x}$ be a committee for object $x \in K$. The subset of agents $M \in \mathcal{W}_{x}$ is a minimal winning coalition on $\mathcal{W}_{x}$ if there is no $M^{\prime} \in \mathcal{W}_{x}$ such that $M^{\prime} \subsetneq M$. Given a committee $\mathcal{W}_{x}$ we denote by $\mathcal{W}_{x}^{m}$ the set of its minimal winning coalitions. 
Assume $f: \mathcal{P}^{n} \rightarrow 2^{K}$ is a voting by committees and let $\mathcal{W}=\left(\mathcal{W}_{x}\right)_{x \in K}$ be its associated family of committees, one for each object. Abusing notation we will often write $f$ directly as $\mathcal{W}: \mathcal{P}^{n} \rightarrow 2^{K}$; hence, for $P \in \mathcal{P}^{n}, \mathcal{W}(P)$ will denote the subset of objects chosen by the voting by committees $\mathcal{W}$ at $P$.

Let $\mathcal{W}$ be a voting by committees. We define three different notions of power that agents may have at $\mathcal{W}$ with respect to their role on the choice of the subset of objects. These notions will be relevant to compare voting by committees according to their manipulability.

First, agent $i$ is dummy at $x$ if $i$ does not belong to any minimal winning coalition on $\mathcal{W}_{x}$; hence $i$ does not play any role on the choice of whether or not $x$ belongs to the chosen set of objects according to $\mathcal{W}$. The set of objects at which agent $i$ is dummy, given $\mathcal{W}$, is defined as

$$
D u_{i}^{\mathcal{W}}=\left\{x \in K \mid \text { for all } S \in \mathcal{W}_{x}^{m}, i \notin S\right\} .
$$

We say that a voting by committees $\mathcal{W}: \mathcal{P}^{n} \rightarrow 2^{K}$ has no dummies if, for all $i \in N$, $D u_{i}^{\mathcal{W}}=\{\emptyset\}$. Without loss of generality we assume that no agent is dummy at all objects. That is, for all $i \in N$,

$$
D u_{i}^{\mathcal{W}} \neq K
$$

otherwise if $D u_{i}^{\mathcal{W}}=K$, then $\mathcal{M}_{i}^{\mathcal{W}}=\{\emptyset\}$ and therefore we may proceed by setting $N:=$ $N \backslash\{i\}$.

Second, agent $i$ is decisive at $x$ if $i$, as a singleton set, belongs to $\mathcal{W}_{x}$; hence, $i$ can impose object $x$ in the chosen subset by declaring it as an element in the top subset of objects. The set of objects at which agent $i$ is decisive, given $\mathcal{W}$, is defined as

$$
D e_{i}^{\mathcal{W}}=\left\{x \in K \mid\{i\} \in \mathcal{W}_{x}\right\}
$$

Third, agent $i$ is a vetoer at $x$ if $i$ belongs to all coalitions on $\mathcal{W}_{x}$; hence, $i$ can veto object $x$ by not declaring it as an element in the top subset of objects. The set of objects at which agent $i$ is vetoer, given $\mathcal{W}$, is defined as

$$
V e_{i}^{\mathcal{W}}=\left\{x \in K \mid i \in \bigcap_{S \in \mathcal{W}_{x}} S\right\}
$$

Example 1 below illustrates how voting by committees work and the three notions of power.

Example 1 Let $N=\{1,2,3,4\}$ be the set of agents and $K=\{x, y, z, w\}$ the set of objects. Consider the voting by committees $\mathcal{W}$ defined by the following (minimal) committees:

$$
\begin{aligned}
& \mathcal{W}_{x}^{m}=\{S \subset N|| S \mid=2\}, \\
& \mathcal{W}_{y}^{m}=\{\{1,2\},\{2,3\}\}, \\
& \mathcal{W}_{z}^{m}=\{\{1,2,3,4\}\}, \text { and } \\
& \mathcal{W}_{w}^{m}=\{\{1\},\{4\}\} .
\end{aligned}
$$


Take any pair of preference profiles $P, P^{\prime} \in \mathcal{P}^{4}$ with the properties that

$$
\begin{aligned}
& t\left(P_{1}\right)=\{x, y\}, t\left(P_{2}\right)=\{y, w\}, t\left(P_{3}\right)=\{x, y\}, \text { and } t\left(P_{4}\right)=\{y, z, w\} \text { and } \\
& t\left(P_{1}^{\prime}\right)=\{y\}, t\left(P_{2}^{\prime}\right)=\{z, w\}, t\left(P_{3}^{\prime}\right)=\{y, z\}, \text { and } t\left(P_{4}^{\prime}\right)=\{w\} .
\end{aligned}
$$

Then, $\mathcal{W}(P)=\{x, y, w\}$ and $\mathcal{W}\left(P^{\prime}\right)=\{w\}$. Observe that the sets related with the power of the agents at $\mathcal{W}$ are:

$$
\begin{aligned}
D u_{1}^{\mathcal{W}} & =\{\emptyset\}, D u_{2}^{\mathcal{W}}=\{w\}, D u_{3}^{\mathcal{W}}=\{w\}, \text { and } D u_{4}^{\mathcal{W}}=\{y\}, \\
D e_{1}^{\mathcal{W}} & =\{w\}, D e_{2}^{\mathcal{W}}=\{\emptyset\}, D e_{3}^{\mathcal{W}}=\{\emptyset\}, \text { and } D e_{4}^{\mathcal{W}}=\{w\}, \text { and } \\
V e_{1}^{\mathcal{W}} & =\{z\}, V e_{2}^{\mathcal{W}}=\{y, z\}, V e_{3}^{\mathcal{W}}=\{z\}, \text { and } V e_{4}^{\mathcal{W}}=\{z\} .
\end{aligned}
$$

Our first preliminary result states that agent $i$ cannot affect the choice of the objects at which $i$ is a dummy agent.

Lemma 1 Let $\mathcal{W}: \mathcal{P}^{n} \rightarrow 2^{K}$ be a voting by committees. Then, for all $\left(P_{i}, P_{-i}\right) \in \mathcal{P}^{n}$ and $P_{i}^{\prime} \in \mathcal{P}$

$$
\mathcal{W}\left(P_{i}, P_{-i}\right) \cap D u_{i}^{\mathcal{W}}=\mathcal{W}\left(P_{i}^{\prime}, P_{-i}\right) \cap D u_{i}^{\mathcal{W}}
$$

Proof Fix $P_{-i} \in \mathcal{P}^{n-1}$. It will be sufficient to show that, for any pair $P_{i}, P_{i}^{\prime} \in \mathcal{P}$, $\mathcal{W}\left(P_{i}, P_{-i}\right) \cap D u_{i}^{\mathcal{W}} \subset \mathcal{W}\left(P_{i}^{\prime}, P_{-i}\right)$. Assume $x \in \mathcal{W}\left(P_{i}, P_{-i}\right) \cap D u_{i}^{\mathcal{W}}$. Then, there exists $S \in \mathcal{W}_{x}^{m}$ such that $x \in t\left(P_{j}\right)$ for all $j \in S$. Since $x \in D u_{i}^{\mathcal{W}}, i \notin S$. Thus, $x \in \mathcal{W}\left(P_{i}^{\prime}, P_{-i}\right)$.

The comparability between two voting by committees in terms of their manipulability will relay strongly on the inclusion relationship between the two induced sets of alternatives that may be selected by them, once the preference of a fixed agent $i$ is given.

Definition 5 Let $f: \mathcal{P}^{n} \rightarrow 2^{K}$ be a social choice function and let $P_{i} \in \mathcal{P}$. The set of options left open by $P_{i} \in \mathcal{P}$ at $f$ is defined as follows:

$$
o^{f}\left(P_{i}\right)=\left\{A \in 2^{K} \mid f\left(P_{i}, P_{-i}\right)=A \text { for some } P_{-i} \in \mathcal{P}^{n-1}\right\} .
$$

Given two subsets of objects $A, B \in 2^{K}$ such that $A \subset B$ let $[A, B]$ be the family of all subsets of objects that can be obtained from $A$ by adding to it objects in $B \backslash A$. Namely, for any pair of alternatives $A \subset B \subset K$,

$$
[A, B]=\{C \subset K \mid A \subset C \subset B\} .
$$

Although imperfectly, to obtain an intuitive geometric idea, the set $[A, B]$ can be seen as the elements in the cone lying between $A$ and $B$, where $\left[\{\emptyset\}, 2^{K}\right]$ would be the cone containing all subsets of $K$ (see Figure 1 below). 


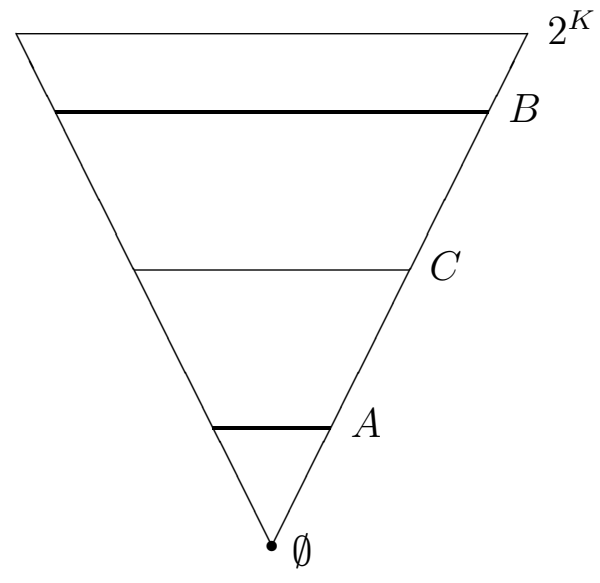

Figure 1

Next lemma characterizes the set of options left open by $P_{i}$ at $\mathcal{W}$ in terms of $t\left(P_{i}\right)$ and the sets of objects at which $i$ is decisive and no vetoer. This result plays a crucial role in the sequel and it will be intensively used. The intuition why it holds is as follows. Fix a preference $P_{i} \in \mathcal{P}$. First, any subset of objects belonging to the set of options left open by $P_{i}$ has to contain the objects in $t\left(P_{i}\right)$ for which $i$ is simultaneously decisive at them; this is so because agent $i$ has voted for them and $i$ has the power to include them. Second, any subset of objects in the set of options left open by $P_{i}$ has to be contained in the set made by the union of the set of objects in $t\left(P_{i}\right)$ and the subset of objects at which $i$ is not a vetoer; this is so because any object for which agent $i$ has not voted for and simultaneously $i$ is a vetoer at will never belong to the chosen subset of objects. Moreover, any subset of objects that does satisfy the two conditions above will belong to the set of option left open by $P_{i}$ at $\mathcal{W}$ because, whenever all remaining agents declare this set as their top subset of objects, it will be selected by $\mathcal{W}$ since the vote of $i$ is not required (because $i$ is not a vetoer) against the unanimous vote of the remaining set of agents. Figure 2 illustrates the set of options left open by $P_{i}$ at $\mathcal{W}$.

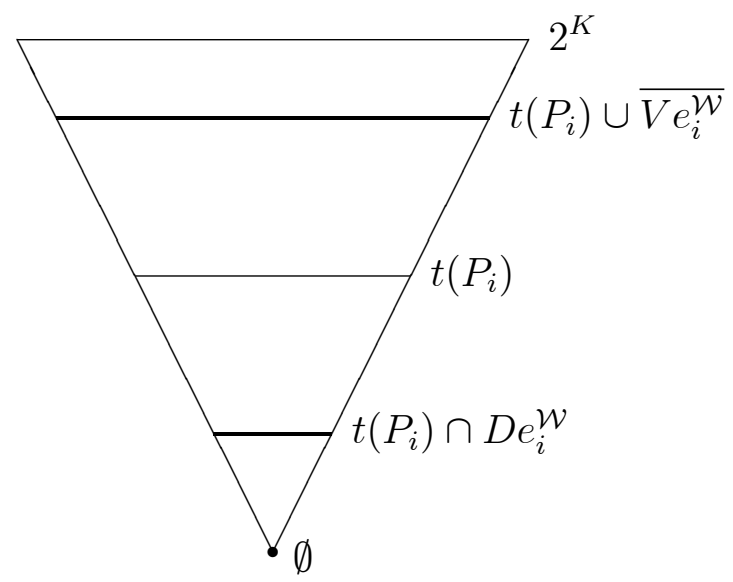

Figure 2 
Lemma 2 Let $\mathcal{W}: \mathcal{P}^{n} \rightarrow 2^{K}$ be a voting by committees. Then, for all $P_{i} \in \mathcal{P}$,

$$
o^{\mathcal{W}}\left(P_{i}\right)=\left[t\left(P_{i}\right) \cap D e_{i}^{\mathcal{W}}, t\left(P_{i}\right) \cup \overline{V e_{i}^{\mathcal{W}}}\right]
$$

Proof Assume $A \in o^{\mathcal{W}}\left(P_{i}\right)$. Then, there exists $P_{-i} \in \mathcal{P}^{n-1}$ such that $A=\mathcal{W}\left(P_{i}, P_{-i}\right)$. Let $x \in t\left(P_{i}\right) \cap D e_{i}^{\mathcal{W}}$. Then, $x \in t\left(P_{i}\right)$ and $\{i\} \in \mathcal{W}_{x}$. Hence, $x \in \mathcal{W}\left(P_{i}, P_{-i}\right)=A$. Thus, $t\left(P_{i}\right) \cap D e_{i}^{\mathcal{W}} \subset A$. Let $x \in A$ and assume that $x \notin t\left(P_{i}\right)$. We will prove that $x \in \overline{V e_{i}^{\mathcal{W}}}$. Since $x \in \mathcal{W}\left(P_{i}, P_{-i}\right)$ and $x \notin t\left(P_{i}\right)$, there exists $S \in \mathcal{W}_{x}^{m}$ such that $S \subset\left\{j \in N \mid x \in t\left(R_{j}\right)\right\}$ and $i \notin S$. Hence, $x \in \overline{V e_{i}^{\mathcal{W}}}$. Thus, $A \subset t\left(P_{i}\right) \cup \overline{V e_{i}^{\mathcal{W}}}$.

Now, let $A \in\left[t\left(P_{i}\right) \cap D e_{i}^{\mathcal{W}}, t\left(P_{i}\right) \cup \overline{V e_{i}^{\mathcal{W}}}\right]$. For each $j \in N \backslash\{i\}$, let $P_{j}$ be any preference such that $t\left(P_{j}\right)=A$. We will prove that $\mathcal{W}\left(P_{i}, P_{-i}\right)=A$. To prove one of the two inclusions, assume $x \in A$. If $x \in t\left(P_{i}\right)$, then $\left\{j \in N \mid x \in t\left(P_{j}\right)\right\}=N$. Hence, $x \in \mathcal{W}\left(P_{i}, P_{-i}\right)$. If $x \notin t\left(P_{i}\right)$, then $x \in \overline{V e_{i}^{\mathcal{W}}}$ (since $A \subset t\left(P_{i}\right) \cup \overline{V e_{i}^{\mathcal{W}}}$ ). Hence, $\left\{j \in N \mid x \in t\left(P_{j}\right)\right\}=N \backslash\{i\} \in \mathcal{W}_{x}$ and $x \in \mathcal{W}\left(P_{i}, P_{-i}\right)$. To prove the other inclusion, assume $x \in \mathcal{W}\left(P_{i}, P_{-i}\right)$. By the definition of $t\left(P_{j}\right)$, either $x \in A$ or else $x \in t\left(P_{i}\right) \backslash A$ and $\{i\} \in \mathcal{W}_{x}$. Hence, either $x \in A$ or else $x \in t\left(P_{i}\right) \cap D e_{i}^{\mathcal{W}}$. Thus, since by assumption $t\left(P_{i}\right) \cap D e_{i}^{\mathcal{W}} \subset A, x \in A$.

In light of Lemma 2 it is easy to see that as the decisive and vetoer sets of objects become larger the option set left open by a preference becomes smaller. Figure 3 illustrates this statement and Lemma 3 states it formally.

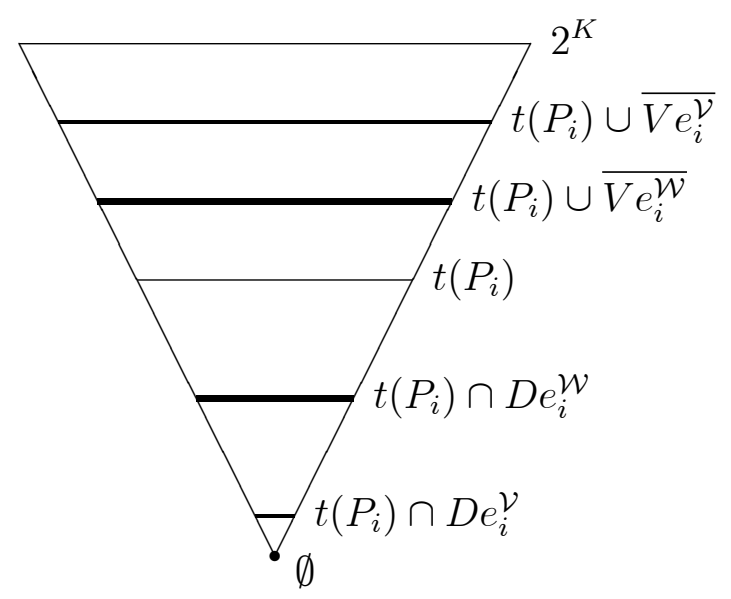

Figure 3

Lemma 3 Let $\mathcal{W}: \mathcal{P}^{n} \rightarrow 2^{K}$ and $\mathcal{V}: \mathcal{P}^{n} \rightarrow 2^{K}$ be two voting by committees with the property that $D e_{i}^{\mathcal{V}} \subset D e_{i}^{\mathcal{W}}$ and $V e_{i}^{\mathcal{V}} \subset V e_{i}^{\mathcal{W}}$. Then, o $o^{\mathcal{W}}\left(P_{i}\right) \subset o^{\mathcal{V}}\left(P_{i}\right)$ for all $P_{i} \in \mathcal{P}$.

Proof It follows immediately from Lemma 2.

In the last preliminary result of this section we identify the necessary and sufficient conditions under which a voting by committees $\mathcal{W}$ is not manipulable by agent $i$ at a particular 
preference $P_{i}$. These conditions can be seen as a weakening of the separability conditions because they require to compare in a specific way fewer pairs of subsets of objects. These pairs are composed of two kinds of sets. First, any subset $A$ that is selected by $\mathcal{W}$ when $i$ votes for $t\left(P_{i}\right)$ (i.e., $A \in o^{\mathcal{W}}\left(P_{i}\right)$ ). Second, any subset that can be obtained from $A$ by taking out objects in $t\left(P_{i}\right)$ at which $i$ is not a dummy and by simultaneously adding objects not in $A$ that are in $\overline{t\left(P_{i}\right)}$ and at which $i$ is not a dummy. Lemma 4 can be seen as providing a general maximal domain result for all voting by committees, which depends on the sets of decisive, vetoers and dummy objects of agent $i$ at $\mathcal{W} .{ }^{3}$ Figure 4 illustrates a particular pair of distinct subsets $\left(A\right.$ and $\left.\left(A \backslash T_{1}\right) \cup T_{2}\right)$ in the cone $\left[\{\emptyset\}, 2^{K}\right]$ that have to be comparable by $P_{i}$ (i.e., $\left.A P_{i}\left(A \backslash T_{1}\right) \cup T_{2}\right)$ to ensure that $\mathcal{W}$ is not manipulable by $i$ at $P_{i}$.

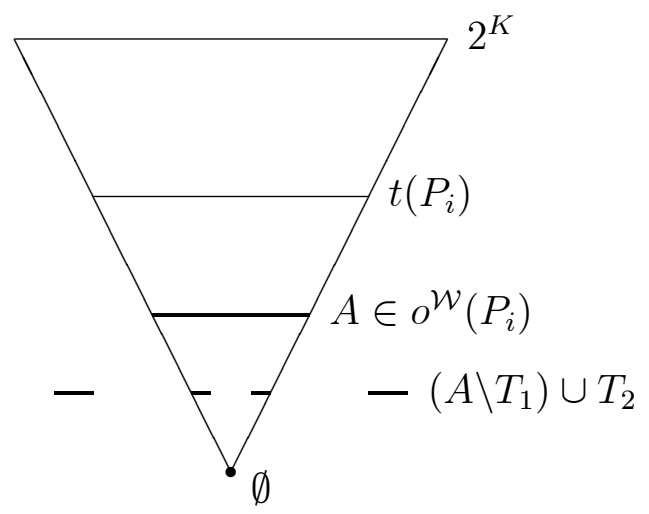

Figure 4

Lemma 4 Let $\mathcal{W}: \mathcal{P}^{n} \rightarrow 2^{K}$ be a voting by committees. Then, $\mathcal{W}$ is not manipulable by $i \in N$ at $P_{i} \in \mathcal{P}$ if and only if for all $A \in o^{\mathcal{W}}\left(P_{i}\right), T_{1} \subset t\left(R_{i}\right) \cap A \cap \overline{D u_{i}^{\mathcal{V}}}$ and $T_{2} \subset$ $\overline{t\left(R_{i}\right)} \cap \bar{A} \cap \overline{D u_{i}^{\mathcal{W}}}$,

$$
A R_{i}\left(A \backslash T_{1}\right) \cup T_{2}
$$

Proof $\Rightarrow)$ Consider any $P_{i} \in \mathcal{P}$ at which $\mathcal{W}$ is not manipulable by $i \in N$ and let $A \in$ $o^{\mathcal{W}}\left(P_{i}\right), T_{1} \subset t\left(P_{i}\right) \cap A \cap \overline{D u_{i}^{\mathcal{W}}}$ and $T_{2} \subset \overline{t\left(P_{i}\right)} \cap \bar{A} \cap \overline{D u_{i}^{\mathcal{W}}}$ be arbitrary. Then, there exists $P_{-i} \in \mathcal{P}^{n-1}$ such that

$$
\mathcal{W}\left(P_{i}, P_{-i}\right)=A \text {. }
$$

\footnotetext{
${ }^{3}$ See Barberà, Massó, and Neme (1999), Barberà, Sonnesnchein, and Zhou (1991) and Serizawa (1995) for related results identifying maximal domains of preferences under which voting by committees remain strategy-proof. The results on the second and third papers are less general since they apply only to voting by committees without vetoer and dummy agents or without dummy agents, respectively. The results in the first paper are presented in the different setting of multidimensional generalized median voters schemes and hence, they are stated in terms of left and right coalition systems instead of committees.
} 
Since $T_{1} \cup T_{2} \subset \overline{D u_{i}^{\mathcal{W}}}$, for each $x \in T_{1} \cup T_{2}$ there is $S_{x}^{i} \in \mathcal{W}_{x}^{m}$ such that $i \in S_{x}^{i}$. For each $j \in N \backslash\{i\}$, consider any $P_{j}^{\prime} \in \mathcal{P}$ such that

$$
t\left(P_{j}^{\prime}\right)=\left(t\left(P_{j}\right) \backslash\left\{x \in T_{1} \cup T_{2} \mid j \notin S_{x}^{i}\right\}\right) \cup\left\{x \in T_{1} \cup T_{2} \mid j \in S_{x}^{i}\right\}
$$

and let $P_{i}^{\prime} \in \mathcal{P}$ be any preference with the property that

$$
t\left(P_{i}^{\prime}\right)=\left(A \backslash T_{1}\right) \cup T_{2}
$$

$\underline{\text { Claim } 1} \mathcal{W}\left(P_{i}, P_{-i}^{\prime}\right)=A$ and $\mathcal{W}\left(P_{i}^{\prime}, P_{-i}^{\prime}\right)=\left(A \backslash T_{1}\right) \cup T_{2}$.

$\underline{\text { Proof of Claim } 1}$ We first show that $\mathcal{W}\left(P_{i}, P_{-i}^{\prime}\right)=A$. Assume $x \in T_{2}$. By $(4),\{j \in N \backslash\{i\} \mid$ $\left.x \in t\left(P_{j}^{\prime}\right)\right\}=S_{x}^{i} \backslash\{i\}$. Hence, and since $T_{2} \subset \overline{t\left(P_{i}\right)}, x \notin t\left(P_{i}\right)$. Observe that $i \in S_{x}^{i} \in \mathcal{W}_{x}^{m}$ implies $S_{x}^{i} \backslash\{i\} \notin \mathcal{W}_{x}$. Thus, $x \notin \mathcal{W}\left(P_{i}, P_{-i}^{\prime}\right)$. Therefore,

$$
\mathcal{W}\left(P_{i}, P_{-i}^{\prime}\right) \cap T_{2}=\{\emptyset\}
$$

Assume $x \in T_{1}$. By (4), $\left\{j \in N \backslash\{i\} \mid x \in t\left(P_{j}^{\prime}\right)\right\}=S_{x}^{i} \backslash\{i\}$. Furthermore, as $T_{1} \subset t\left(P_{i}\right)$, $x \in t\left(R_{i}\right)$. Observe that $S_{x}^{i} \in \mathcal{W}_{x}^{m}$. Thus, $x \in \mathcal{W}\left(P_{i}, P_{-i}^{\prime}\right)$. Therefore,

$$
\mathcal{W}\left(P_{i}, P_{-i}^{\prime}\right) \cap T_{1}=T_{1}
$$

Assume $x \notin T_{1} \cup T_{2}$. By (4), $\left\{j \in N \backslash\{i\} \mid x \in t\left(P_{j}^{\prime}\right)\right\}=\left\{j \in N \backslash\{i\} \mid x \in t\left(P_{j}\right)\right\}$. Hence,

$$
\left(\overline{T_{1} \cup T_{2}}\right) \cap \mathcal{W}\left(P_{i}, P_{-i}^{\prime}\right)=\left(\overline{T_{1} \cup T_{2}}\right) \cap \mathcal{W}\left(P_{i}, P_{-i}\right)
$$

By (5), (6) and (7),

$$
\begin{aligned}
\mathcal{W}\left(P_{i}, P_{-i}^{\prime}\right) & =\left[\left(\overline{T_{1} \cup T_{2}}\right) \cap \mathcal{W}\left(P_{i}, P_{-i}^{\prime}\right)\right] \cup T_{1} \\
& =\left[\left(\overline{T_{1} \cup T_{2}}\right) \cap \mathcal{W}\left(P_{i}, P_{-i}\right)\right] \cup T_{1} \\
& =\left[\left(\overline{T_{1} \cup T_{2}}\right) \cap A\right] \cup T_{1} \\
& =A,
\end{aligned}
$$

where the last equality follows from the facts that $T_{1} \subset A$ and $T_{2} \subset \bar{A}$.

We now show that $\mathcal{W}\left(P_{i}^{\prime}, P_{-i}^{\prime}\right)=\left(A \backslash T_{1}\right) \cup T_{2}$. Assume $x \in A \backslash T_{1}$. If $x \in t\left(P_{i}\right)$, and since $\mathcal{W}\left(P_{i}, P_{-i}^{\prime}\right)=A,\left\{j \in N \backslash\{i\} \mid x \in t\left(P_{j}^{\prime}\right)\right\} \cup\{i\} \in \mathcal{W}_{x}$ holds. If $x \notin t\left(P_{i}\right)$, and since $\mathcal{W}\left(P_{i}, P_{-i}^{\prime}\right)=A,\left\{j \in N \backslash\{i\} \mid x \in t\left(P_{j}^{\prime}\right)\right\} \in \mathcal{W}_{x}$ holds. Hence, in both cases,

$$
\left\{j \in N \backslash\{i\} \mid x \in t\left(P_{j}^{\prime}\right)\right\} \cup\{i\} \in \mathcal{W}_{x} .
$$

Since $x \in t\left(P_{i}^{\prime}\right),\left\{j \in N \backslash\{i\} \mid x \in t\left(P_{j}^{\prime}\right)\right\} \cup\{i\}=\left\{j \in N \mid x \in t\left(P_{j}^{\prime}\right)\right\}$. Hence, by (8), $\left\{j \in N \mid x \in t\left(P_{j}^{\prime}\right)\right\} \in \mathcal{W}_{x}$. Thus, $x \in \mathcal{W}\left(P_{i}^{\prime}, P_{-i}^{\prime}\right)$. Assume now $x \in T_{2}$. By (4), $\left\{j \in N \backslash\{i\} \mid x \in t\left(P_{j}^{\prime}\right)\right\}=S_{x}^{i} \backslash\{i\}$. Hence, and since $x \in t\left(P_{i}^{\prime}\right), x \in \mathcal{W}\left(P_{i}^{\prime}, P_{-i}^{\prime}\right)$. Therefore, 
we have showed that $\left(A \backslash T_{1}\right) \cup T_{2} \subset \mathcal{W}\left(P_{i}^{\prime}, P_{-i}^{\prime}\right)$. To show that the other inclusion holds, assume $x \in \mathcal{W}\left(P_{i}^{\prime}, P_{-i}^{\prime}\right)$. If $x \in t\left(P_{i}^{\prime}\right)$, by the definition of $t\left(P_{i}^{\prime}\right), x \in\left(A \backslash T_{1}\right) \cup T_{2}$. If, on the other hand, $x \notin t\left(P_{i}^{\prime}\right)$,

$$
\left\{j \in N \mid x \in t\left(P_{j}^{\prime}\right)\right\}=\left\{j \in N \backslash\{i\} \mid x \in t\left(P_{j}^{\prime}\right)\right\} .
$$

Hence, and since $x \in \mathcal{W}\left(P_{i}^{\prime}, P_{-i}^{\prime}\right)$, by $(9), x \in \mathcal{W}\left(P_{i}, P_{-i}^{\prime}\right)=A$. Now, to obtain a contradiction assume $x \in T_{1}$. Hence, $\left\{j \in N \backslash\{i\} \mid x \in t\left(P_{j}^{\prime}\right)\right\}=S_{x}^{i} \backslash\{i\}$ and $x \notin t\left(P_{i}^{\prime}\right)$. Therefore, $x \notin \mathcal{W}\left(P_{i}^{\prime}, P_{-i}^{\prime}\right)$ which is a contradiction. Thus, $x \in A \backslash T_{1}$.

Therefore, by Claim 1 and the fact that $\mathcal{W}$ is not manipulable by $i$ at $P_{i}$,

$$
A R_{i}\left(A \backslash T_{1}\right) \cup T_{2}
$$

$\Leftarrow)$ Let $P_{i} \in \mathcal{P}$ be arbitrary and assume that for all $A \in o^{\mathcal{W}}\left(P_{i}\right), T_{1} \subset t\left(R_{i}\right) \cap A \cap \overline{D u_{i}^{\mathcal{V}}}$ and $T_{2} \subset \overline{t\left(R_{i}\right)} \cap \bar{A} \cap \overline{D u_{i}^{\mathcal{W}}}, A R_{i}\left(A \backslash T_{1}\right) \cup T_{2}$ holds. We will show that, for all $\left(P_{i}^{\prime}, P_{-i}\right) \in \mathcal{P}^{n}$,

$$
\mathcal{W}\left(P_{i}, P_{-i}\right) R_{i} \mathcal{W}\left(P_{i}^{\prime}, P_{-i}\right)
$$

Assume $\left(P_{i}^{\prime}, P_{-i}\right) \in \mathcal{P}^{n}$ and let $\mathcal{W}\left(P_{i}, P_{-i}\right)=A \in o^{\mathcal{W}}\left(P_{i}\right), T_{1}=\left(A \backslash \mathcal{W}\left(P_{i}^{\prime}, P_{-i}\right)\right) \cap \overline{D u_{i}^{\mathcal{W}}}$ and $T_{2}=\left(\mathcal{W}\left(P_{i}^{\prime}, P_{-i}\right) \backslash A\right) \cap \overline{D u_{i}^{\mathcal{W}}}$. Then,

$$
\begin{array}{rlr}
\left(A \backslash T_{1}\right) \cup T_{2} & =\left[\left(A \cap D u_{i}^{\mathcal{W}} \cup A \cap \overline{D u_{i}^{\mathcal{W}}}\right) \backslash T_{1}\right] \cup T_{2} & \\
& =\left(A \cap D u_{i}^{\mathcal{W}}\right) \cup\left[\left(A \cap \overline{D u_{i}^{\mathcal{W}}}\right) \backslash T_{1}\right] \cup T_{2} & \text { since } T_{1} \subset \overline{D u_{i}^{\mathcal{W}}} \\
& =\left(A \cap D u_{i}^{\mathcal{W}}\right) \cup\left[\left(A \cap \overline{D u_{i}^{\mathcal{W}}}\right) \cap \overline{T_{1}}\right] \cup T_{2} \\
& =\left(A \cap D u_{i}^{\mathcal{W}}\right) \cup\left[\left(A \cap \overline{D u_{i}^{\mathcal{W}}}\right) \cap\left(\bar{A} \cup \mathcal{W}\left(P_{i}^{\prime}, P_{-i}\right) \cup D u_{i}^{\mathcal{W}}\right)\right] \cup T_{2} \\
& =\left(A \cap D u_{i}^{\mathcal{W}}\right) \cup\left[\left(A \cap \overline{D u_{i}^{\mathcal{W}}}\right) \cap \mathcal{W}\left(P_{i}^{\prime}, P_{-i}\right)\right] \cup T_{2} \\
& \left.=\left(A \cap D u_{i}^{\mathcal{W}}\right) \cup\left[\left(A \cap \overline{D u_{i}^{\mathcal{W}}}\right) \cap \mathcal{W}\left(P_{i}^{\prime}, P_{-i}\right)\right] \cup\left(\mathcal{W}\left(P_{i}^{\prime}, P_{-i}\right) \backslash A\right) \cap \overline{D u_{i}^{\mathcal{W}}}\right) \\
& =\left(A \cap D u_{i}^{\mathcal{W}}\right) \cup\left[\left(A \cap \mathcal{W}\left(P_{i}^{\prime}, P_{-i}\right) \cup\left(\mathcal{W}\left(P_{i}^{\prime}, P_{-i}\right) \backslash A\right)\right] \cap \overline{D u_{i}^{\mathcal{W}}}\right. \\
& =\left(A \cap D u_{i}^{\mathcal{W}}\right) \cup\left(\mathcal{W}\left(P_{i}^{\prime}, P_{-i}\right) \cap \overline{D u_{i}^{\mathcal{W}}}\right) \\
& =\left(\mathcal{W}\left(P_{i}^{\prime}, P_{-i}\right) \cap D u_{i}^{\mathcal{W}}\right) \cup\left(\mathcal{W}\left(P_{i}^{\prime}, P_{-i}\right) \cap \overline{D u_{i}^{\mathcal{W}}}\right) \\
& =\mathcal{W}\left(P_{i}^{\prime}, P_{-i}\right) .
\end{array}
$$

Furthermore, $T_{1} \subset t\left(R_{i}\right) \cap A \cap \overline{D u_{i}^{\mathcal{W}}}$ and $T_{2} \subset \overline{t\left(R_{i}\right)} \cap \bar{A} \cap \overline{D u_{i}^{\mathcal{V}}}$ since $A \backslash \mathcal{W}\left(P_{i}^{\prime}, P_{-i}\right) \subset t\left(R_{i}\right)$ and $\mathcal{W}\left(P_{i}^{\prime}, P_{-i}\right) \backslash A \subset \overline{t\left(P_{i}\right)}$. Thus, by hypothesis,

$$
\mathcal{W}\left(P_{i}, P_{-i}\right)=A R_{i}\left(A \backslash T_{1}\right) \cup T_{2}=\mathcal{W}\left(P_{i}^{\prime}, P_{-i}\right)
$$




\section{Comparing Voting by Committees without dummies}

\subsection{Main Result Without Dummies}

Theorem 1 below gives an easy and operative way to compare voting by committees without dummies according to their manipulability. A voting by committees $\mathcal{V}$ is at least as manipulable as voting by committees $\mathcal{W}$ if and only if, for each agent $i \in N$, the sets of objects at which agent $i$ is decisive and vetoer in $\mathcal{V}$ is each contained in the corresponding sets in $\mathcal{W}$. The results in the preceding lemmata are key to understand this characterization. Larger decisive and vetoer sets of objects make the option sets left open smaller and this leaves more freedom on the comparability between subsets of objects, reducing hence the set of preferences under which the agent is able to manipulate.

Theorem 1 Let $\mathcal{V}: \mathcal{P}^{n} \rightarrow 2^{K}$ and $\mathcal{W}: \mathcal{P}^{n} \rightarrow 2^{K}$ be two voting by committees without dummies. Then, $\mathcal{V} \succsim \mathcal{W}$ if and only if, for all $i \in N, D e_{i}^{\mathcal{V}} \subset D e_{i}^{\mathcal{W}}$ and $V e_{i}^{\mathcal{V}} \subset V e_{i}^{\mathcal{W}}$.

Proof $\Leftarrow)$ To prove sufficiency, assume that for all $i \in N, D e_{i}^{\mathcal{V}} \subset D e_{i}^{\mathcal{W}}$ and $V e_{i}^{\mathcal{V}} \subset V e_{i}^{\mathcal{W}}$. We prove that $\mathcal{V} \succsim \mathcal{W}$ by showing that, for all $i \in N, \mathcal{M}_{i}^{\mathcal{W}} \subset \mathcal{M}_{i}^{\mathcal{V}}$. Suppose $P_{i} \in \mathcal{M}_{i}^{\mathcal{W}}$. Observe that since $\mathcal{V}$ and $\mathcal{W}$ have no dummies, $\overline{D u_{i}^{\mathcal{W}}}=\overline{D u_{i}^{\mathcal{V}}}=K$ holds. Then, by Lemma 4, there exist $A \in o^{\mathcal{W}}\left(P_{i}\right), T_{1} \subset t\left(P_{i}\right) \cap A$ and $T_{2} \subset \overline{t\left(P_{i}\right)} \cap \bar{A}$ such that

$$
\left(A \backslash T_{1}\right) \cup T_{2} P_{i} A .
$$

By Lemma $3, o^{\mathcal{W}}\left(P_{i}\right) \subset o^{\mathcal{V}}\left(P_{i}\right)$. Hence, $A \in o^{\mathcal{V}}\left(P_{i}\right)$. Thus, by Lemma $4, P_{i} \in \mathcal{M}_{i}^{\mathcal{V}}$. Therefore, $\mathcal{V}$ is at least as manipulable as $\mathcal{W}$.

$\Rightarrow$ ) To prove necessity assume that $\mathcal{V}$ is at least as manipulable as $\mathcal{W}$. Hence, for all $i \in N$,

$$
\mathcal{M}_{i}^{\mathcal{W}} \subset \mathcal{M}_{i}^{\mathcal{V}}
$$

Assume, to obtain a contradiction, that there exists $x \in K$ such that $x \in V e_{i}^{\mathcal{V}} \backslash V e_{i}^{\mathcal{W}}$ or $x \in D e_{i}^{\mathcal{V}} \backslash D e_{i}^{\mathcal{W}}$. We distinguish between these two cases.

Case 1: There exists $x \in V e_{i}^{\mathcal{V}} \backslash V e_{i}^{\mathcal{W}}$. Consider any $P_{i}^{\prime} \in \mathcal{P}$ such that $t\left(P_{i}^{\prime}\right)=\{\emptyset\}$ satisfying in addition the following properties:

(i) $B P_{i}^{\prime} C P_{i}^{\prime}\{x\}$ if $x \notin B$ and $x \in C$,

(ii) $A R_{i}^{\prime} B$ if $A \subset B$ and $x \notin B$.

Three comments on the preference $P_{i}^{\prime}$ are appropriate. First, such preference exists and, because we assumed that $|K| \geq 2$, there exists $C \subset K$ such that $x \in C$ and $\{x\} \neq C$. Second, any pair of subsets of objects that are unrestricted by conditions (i) and (ii) can be ordered arbitrarily by $P_{i}^{\prime}$. Third, $P_{i}^{\prime}$ is not separable since $K P_{i}^{\prime}\{x\}$ while all objects are bad. 
Since $x \in V e_{i}^{\mathcal{V}} \backslash V e_{i}^{\mathcal{W}},\{x\} \in o^{\mathcal{W}}\left(P_{i}^{\prime}\right)$. Set $A=\{x\}, T_{1}=\{\emptyset\}$ and $T_{2}=C \backslash\{x\}$ where $C$ is such that $x \in C$ and $\{x\} \neq C$ (which exists because $|K| \geq 2$ ). Observe first that $A \in o^{\mathcal{W}}\left(P_{i}^{\prime}\right)$ and, by (i) in the definition of $P_{i}^{\prime},\left(A \backslash T_{1}\right) \cup T_{2}=C P_{i}^{\prime}\{x\}=A$. Hence, by Lemma $4, P_{i}^{\prime} \in \mathcal{M}_{i}^{\mathcal{W}}$. Second, take any $B \in o^{\mathcal{V}}\left(P_{i}^{\prime}\right)$. Since $x \in V e_{i}^{\mathcal{V}}$ and $x \notin t\left(P_{i}^{\prime}\right), x \notin B$. Let $T_{2} \subset \bar{A}$ be arbitrary. By (i) and (ii) in the definition of $P_{i}^{\prime}, B R_{i}^{\prime} B \cup T_{2}$. Hence, by Lemma 4, $P_{i}^{\prime} \notin \mathcal{M}_{i}^{\mathcal{V}}$. Thus, $P_{i}^{\prime} \in \mathcal{M}_{i}^{\mathcal{W}} \backslash \mathcal{M}_{i}^{\mathcal{V}}$ holds, which contradicts (10).

Case 2: There exists $x \in D e_{i}^{\mathcal{V}} \backslash D e_{i}^{\mathcal{W}}$. Consider any $P_{i}^{\prime \prime} \in \mathcal{M}_{i}^{\mathcal{W}}$ such that $t\left(P_{i}^{\prime \prime}\right)=K$ satisfying in addition the following properties:

(i) $B P_{i}^{\prime \prime} C P_{i}^{\prime \prime} K \backslash\{x\}$ if $x \in B$ and $x \notin C$,

(ii) $A R_{i}^{\prime \prime} B$ if $B \subset A$ and $x \in B$.

Three comments on the preference $P_{i}^{\prime \prime}$ are also appropriate. First, such preference exists and because we assumed that $|K| \geq 2$, there exists $C \subset K$ such that $x \notin C$ and $C \neq\{\emptyset\}$. Second, any pair of subsets of objects that are unrestricted by conditions (i) and (ii) can be ordered arbitrarily by $P_{i}^{\prime \prime}$. Third, $P_{i}^{\prime \prime}$ is not separable since $\{\emptyset\} P_{i}^{\prime \prime} K \backslash\{x\}$ while all objects are good.

Since $x \in D e_{i}^{\mathcal{V}} \backslash D e_{i}^{\mathcal{W}}, K \backslash\{x\} \in o^{\mathcal{W}}\left(P_{i}^{\prime \prime}\right)$. Set first $A=K \backslash\{x\}, T_{2}=\{\emptyset\}$ and $T_{1}=C$, where $C$ is such that $x \notin C$ and $C \neq\{\emptyset\}$. Observe that $A \in o^{\mathcal{W}}\left(P_{i}^{\prime \prime}\right), x \notin\left(A \backslash T_{1}\right) \cup T_{2}$ and $\left(A \backslash T_{1}\right) \cup T_{2} \neq\{\emptyset\}$. Then, by (i) in the definition of $P_{i}^{\prime \prime},\left(A \backslash T_{1}\right) \cup T_{2} P_{i}^{\prime \prime} K \backslash\{x\}=A$. Hence, by Lemma $4, P_{i}^{\prime \prime} \in \mathcal{M}_{i}^{\mathcal{W}}$. Second, take any $B \in o^{\mathcal{V}}\left(P_{i}^{\prime \prime}\right)$. Since $x \in D e_{i}^{\mathcal{V}}$ and $x \in t\left(P_{i}^{\prime \prime}\right)$, $x \in B$. Let $T_{1} \subset B$ be arbitrary. By (i) and (ii) in the definition of $P_{i}^{\prime \prime}, B R_{i}^{\prime \prime} B \backslash T_{1}$. Hence, by Lemma $4, P_{i}^{\prime \prime} \notin \mathcal{M}_{i}^{\mathcal{V}}$. Thus, $P_{i}^{\prime \prime} \in \mathcal{M}_{i}^{\mathcal{W}} \backslash \mathcal{M}_{i}^{\mathcal{V}}$ holds, which contradicts (10).

Next proposition identifies those voting by committees without dummies that are less manipulable. They have the properties that (i) the set of objects at which all agents are not vetoers is a subset of the set of decisive objects of each agent, and (ii) the set of objects at which each agent is not a vetoer is contained in the set of objects at which some agent is decisive. But before stating Proposition 2 we present a simple remark that will be used in its proof.

Remark 2 Assume $\mathcal{W}: \mathcal{P}^{n} \rightarrow 2^{K}$ is a voting by committees without dummies. Then, for all $i \in N, D e_{i}^{\mathcal{W}} \subset \overline{V e_{i}^{\mathcal{W}}}$.

To see why Remark 2 holds, assume there exists $x \in D e_{i}^{\mathcal{W}}$ such that $x \in V e_{i}^{\mathcal{W}}$. Then, $\mathcal{W}_{x}^{m}=\{\{i\}\}$. Hence, each $j \neq i$ is a dummy at $x$, which is a contradiction.

Proposition 2 Let $\mathcal{W}: \mathcal{P}^{n} \rightarrow 2^{K}$ be a voting by committees without dummies. Then, there does not exist a voting by committee without dummies $\mathcal{V}: \mathcal{P}^{n} \rightarrow 2^{K}$ such that $\mathcal{W} \succ \mathcal{V}$ if and only if

$$
\cap_{i \in N} \overline{V e_{i}^{\mathcal{W}}} \subset D e_{j}^{\mathcal{W}} \text { for all } j \in N
$$




$$
\overline{V e_{j}^{\mathcal{W}}} \subset \cup_{i \in N} D e_{i}^{\mathcal{W}} \text { for all } j \in N
$$

Proof $\Rightarrow)$ We distinguish between two cases.

Case 1: There exist $j \in N$ and $x \in K$ such that $x \in \cap_{i \in N} \overline{V e_{i}^{\mathcal{W}}}$ and $x \notin D e_{j}^{\mathcal{W}}$. Define $\mathcal{V}$ as follows:

$$
\mathcal{V}_{y}^{m}= \begin{cases}\mathcal{W}_{y}^{m} & \text { if } y \neq x \\ \left\{S \in \mathcal{W}_{x}^{m} \mid j \notin S\right\} \cup\left\{S \backslash\{j\} \subset N \mid S \in \mathcal{W}_{x}^{m} \text { s.t. } j \in S\right\} \cup\{\{j\}\} & \text { if } y=x .\end{cases}
$$

Since $x \notin D e_{j}^{\mathcal{W}}$ and $\mathcal{W}$ does not have dummies, $\{\emptyset\} \notin\left\{S \backslash\{j\} \subset N \mid S \in \mathcal{W}_{x}^{m}\right.$ such that $j \in S\}$ and $\mathcal{V}$ is a well defined voting by committees. Now, we prove that $\mathcal{V}$ is a voting by committees without dummies. For each object $y \neq x$ it is immediate to see that $\mathcal{V}_{y}$ has no dummies since $\mathcal{W}_{y}$ does not have any. We prove that no agent is a dummy at $x$ at $\mathcal{V}$. Let $i \in N$. If $i=j$, it is immediate to see that $i$ is not dummy at $x$ since $\{i\} \in \mathcal{V}_{x}^{m}$ by definition. If $i \neq j$, there exists $S \in \mathcal{W}_{x}^{m}$ such that $i \in S$ (since $\mathcal{W}$ is a voting by committees without dummies). Hence, $S \backslash\{j\} \in \mathcal{V}_{x}^{m}$ and $i$ is not dummy at $x$ at $\mathcal{V}$. Now, we prove that $\mathcal{W} \succ \mathcal{V}$. By Theorem 1, it is sufficient to prove that $D e_{i}^{\mathcal{W}} \subset D e_{i}^{\mathcal{V}}$ and $V e_{i}^{\mathcal{W}} \subset V e_{i}^{\mathcal{V}}$ for all $i \in N$ and that $D e_{j}^{\mathcal{V}} \neq D e_{j}^{\mathcal{W}}$. The last inequality follows from the fact that $x \in D e_{j}^{\mathcal{V}}$ and $x \notin D e_{j}^{\mathcal{W}}$. Then, we only have to show that $D e_{i}^{\mathcal{W}} \subset D e_{i}^{\mathcal{V}}$ and $V e_{i}^{\mathcal{W}} \subset V e_{i}^{\mathcal{V}}$ for all $i \in N$.

Assume $y \in D e_{i}^{\mathcal{W}}$. Then, $\{i\} \in \mathcal{W}_{y}^{m}$, Hence, by definition of $\mathcal{V}_{y}^{m},\{i\} \in \mathcal{V}_{y}^{m}$. Hence, $y \in D e_{i}^{\mathcal{V}}$.

Assume $y \in V e_{i}^{\mathcal{W}}$. Then, $i \in \cap_{S \in \mathcal{W}_{y}^{m}} S$. Since $y \in V e_{i}^{\mathcal{W}}$, and $x \in \cap_{i \in N} \overline{V e_{i}^{\mathcal{W}}}$ holds, $y \neq x$. Hence, by definition of $\mathcal{V}_{y}^{m}, j \in \cap_{S \in \mathcal{V}_{y}^{m}} S$ and $y \in V e_{i}^{\mathcal{V}}$.

Case 2: Assume that there exist $j \in N$ and $x \in K$ such that $x \in \overline{V e_{j}^{\mathcal{W}}}$ and $x \notin \cup_{i \in N} D e_{i}^{\mathcal{W}}$. To define $\mathcal{V}$ set $S^{\prime}=\cap_{S \in \mathcal{W}_{x}^{m}} S$ and consider any $i \neq j$. Define the collection of subsets of agents $\mathcal{T}_{i}$ associated to $i$ as follows:

$$
T \in \mathcal{T}_{i} \text { if and only if } i \notin S^{\prime} \text { and } T=\{i, j\} \cup S^{\prime}
$$

Define now $\mathcal{V}$ by setting

$$
\mathcal{V}_{y}^{m}= \begin{cases}\mathcal{W}_{y}^{m} & \text { if } y \neq x \\ \left\{\mathcal{T}_{i}\right\}_{i \neq j} & \text { if } y=x\end{cases}
$$

Since $x \in \overline{V e_{j}^{\mathcal{W}}}, j \notin S^{\prime}$. Therefore, $\mathcal{V}_{x}^{m}$ is minimal. Furthermore, as $x \in \overline{V e_{j}^{\mathcal{W}}}$ and $\mathcal{W}$ is a voting by committees without dummies, there exists $S \varsubsetneqq N$ such that $j \in S \in \mathcal{W}_{x}^{m}$. Therefore, $S^{\prime} \varsubsetneqq N \backslash\{j\}$. Hence, $\mathcal{V}_{x}^{m} \neq\{\emptyset\}$ and $\mathcal{V}$ is a well defined voting by committees. Moreover, $\mathcal{V}_{y}$ is a committee without dummies because $\mathcal{W}_{y}$ is a committee without dummies at any $y \neq x$. Furthermore, by its definition, $\mathcal{V}_{x}$ is a committee without dummies. Then, $\mathcal{V}$ is a voting by committees without dummies. Now, we prove that $\mathcal{W} \succ \mathcal{V}$. By Theorem 1 , it 
is sufficient to show that $D e_{i}^{\mathcal{W}} \subset D e_{i}^{\mathcal{V}}$ and $V e_{i}^{\mathcal{W}} \subset V e_{i}^{\mathcal{V}}$ for all $i \in N$ and that $V e_{j}^{\mathcal{W}} \neq V e_{j}^{\mathcal{V}}$. The last inequality follows from the fact that $x \notin V e_{j}^{\mathcal{W}}$ and $x \in V e_{j}^{\mathcal{V}}$. Hence, we only have to show that $D e_{i}^{\mathcal{W}} \subset D e_{i}^{\mathcal{V}}$ and $V e_{i}^{\mathcal{W}} \subset V e_{i}^{\mathcal{V}}$ for all $i \in N$.

Assume $y \in D e_{i}^{\mathcal{W}}$. Then, $\{i\} \in \mathcal{W}_{y}^{m}$ and, since $x \notin \cup_{i \in N} D e_{i}^{\mathcal{W}}, y \neq x$. Hence, by the definition of $\mathcal{V}_{y}^{m},\{i\} \in \mathcal{V}_{y}^{m}$. Thus, $y \in D e_{i}^{\mathcal{V}}$.

Assume $y \in V e_{i}^{\mathcal{W}}$ and $y \neq x$. Hence, by the definition of $\mathcal{V}_{y}^{m}, y \in V e_{i}^{\mathcal{V}}$.

Now, assume $x \in V e_{i}^{\mathcal{W}}$. Then, $i \in \cap_{S \in \mathcal{W}_{x}^{m}} S=S^{\prime}$. Then, by definition of $\mathcal{V}_{x}^{m}, x \in V e_{i}^{\mathcal{V}}$.

$\Leftarrow$ ) Assume (11) and (12) hold and let $\mathcal{V}: \mathcal{P}^{n} \rightarrow 2^{K}$ be a voting by committees without dummies such that $\mathcal{W} \succeq \mathcal{V}$. By Theorem $1, D e_{i}^{\mathcal{W}} \subset D e_{i}^{\mathcal{V}}$ and $V e_{i}^{\mathcal{W}} \subset V e_{i}^{\mathcal{V}}$ for all $i \in N$. We prove that $D e_{i}^{\mathcal{W}}=D e_{i}^{\mathcal{V}}$ and $V e^{\mathcal{W}}=V e_{i}^{\mathcal{V}}$ for all $i \in N$ and hence, $\mathcal{W} \nsucc \mathcal{V}$.

Assume there exist $j \in N$ and $x \in K$ such that $x \in D e_{j}^{\mathcal{V}} \backslash D e_{j}^{\mathcal{W}}$. By Remark $2, x \in$ $\overline{V e_{j}^{\mathcal{V}}} \backslash D e_{j}^{\mathcal{W}}$. Since $V e_{j}^{\mathcal{W}} \subset V e_{j}^{\mathcal{V}}, x \in \overline{V e_{j}^{\mathcal{W}}} \backslash D e_{j}^{\mathcal{W}}$. By (11), there exists $i \neq j$ such that $x \in V e_{i}^{\mathcal{W}}$. Thus, $x \in V e_{i}^{\mathcal{V}}$ which contradicts that $x \in D e_{j}^{\mathcal{V}}$ and $i \neq j$ since $i \notin\{j\} \in \mathcal{V}_{x}^{m}$.

Now, assume there exist $j \in N$ and $x \in K$ such that $x \in V e_{j}^{\mathcal{V}} \backslash V e_{j}^{\mathcal{W}}$. Then, $x \in$

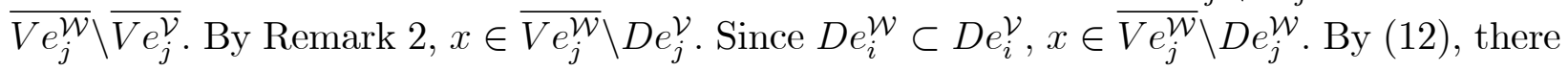
exists $i \neq j$ such that $x \in D e_{i}^{\mathcal{W}}$. Thus, $x \in D e_{i}^{\mathcal{V}}$ which contradicts that $x \in V e_{j}^{\mathcal{V}}$ and $i \neq j$ since $j \notin\{i\} \in \mathcal{V}_{x}^{m}$.

\subsection{Anonymity}

In this subsection we restrict further the class of voting by committees without dummies by requiring that they are indeed anonymous (and hence, no agent is dummy at any object). We show that the binary relation arising from our comparability criterion endows the (quotient) set of anonymous voting by committees with a complete upper semilattice structure that we fully identify.

A social choice function $f: \widehat{\mathcal{P}}^{n} \rightarrow 2^{K}$ is anonymous if it is invariant with respect to the agents' names; namely, for all one-to-one $\sigma: N \rightarrow N$ and all $P \in \widehat{\mathcal{P}}^{n}, f\left(P_{1}, \ldots, P_{n}\right)=$ $f\left(P_{\sigma(1)}, \ldots, P_{\sigma(n)}\right)$.

Remark 3 Let $\mathcal{W}: \mathcal{P}^{n} \rightarrow 2^{K}$ be an anonymous voting by committees. Then, for all $x \in K$, $\mathcal{W}_{x}$ does not have dummy agents. ${ }^{4}$

A voting by committees $\mathcal{W}: \mathcal{P}^{n} \rightarrow 2^{K}$ is a voting by quotas if for each object $x \in K$ there exists an integer $q_{x}^{\mathcal{W}}$ between 1 and $n$ such that

$$
\mathcal{W}_{x}=\left\{S \subset N|| S \mid \geq q_{x}^{\mathcal{W}}\right\}
$$

\footnotetext{
${ }^{4}$ To see that, assume $i$ is dummy at $x$. Then, by anonymity, $j$ must be dummy at $x$ for all $j \in N$. Hence, $\mathcal{W}_{x}=\{\emptyset\}$ which is a contradiction.
} 
Observe that Barberà, Sonnenschein, and Zhou (1991) call voting by quota to a voting by quotas that is in addition neutral in the sense that $q_{x}^{\mathcal{W}}=q_{y}^{\mathcal{W}}$ for all $x, y \in K$. The following remark states that the subclass of anonymous voting by committees coincides with all voting by quotas (not necessarily neutral).

Remark 4 A voting by committees is anonymous if and only if it is voting by quotas.

Let $\mathcal{W}: \mathcal{P}^{n} \rightarrow 2^{K}$ be a voting by quotas. Denote by $A_{1}^{\mathcal{W}}$ and $A_{n}^{\mathcal{W}}$ the set of objects in $K$ that have quota 1 and quota $n$, respectively; namely, $A_{1}^{\mathcal{W}}=\left\{x \in K \mid q_{x}^{\mathcal{W}}=1\right\}$ and $A_{n}^{\mathcal{W}}=\left\{x \in K \mid q_{x}^{\mathcal{W}}=n\right\}$. Since for all voting by quotas $\mathcal{W}: \mathcal{P}^{n} \rightarrow 2^{K}$ it holds that $D e_{i}^{\mathcal{W}}=A_{1}^{\mathcal{W}}$ and $V e_{i}^{\mathcal{W}}=A_{n}^{\mathcal{W}}$ for all $i \in N$, we obtain as a consequence of Theorem 1 the following Corollary.

Corollary 1 Let $\mathcal{W}: \mathcal{P}^{n} \rightarrow 2^{K}$ and $\mathcal{V}: \mathcal{P}^{n} \rightarrow 2^{K}$ be two voting by quotas. Then, $\mathcal{V} \succsim \mathcal{W}$ if and only if $A_{1}^{\mathcal{V}} \subset A_{1}^{\mathcal{W}}$ and $A_{n}^{\mathcal{V}} \subset A_{n}^{\mathcal{W}}$.

The next corollary identifies the class of anonymous voting by committees that do not admit a less manipulable anonymous voting by committees. This class is the family of all voting by quotas such that the quota of each object is either 1 or $n$.

Corollary 2 Let $\mathcal{W}: \mathcal{P}^{n} \rightarrow 2^{K}$ be a voting by quotas. Then, there does not exist a voting by quotas (i.e., an anonymous voting by committees) $\mathcal{V}: \mathcal{P}^{n} \rightarrow 2^{K}$ such that $\mathcal{W} \succ \mathcal{V}$ if and only if $A_{n}^{\mathcal{W}}=K \backslash A_{1}^{\mathcal{W}}$.

Let $V b Q$ be the family of all voting by quotas relative to a given set of agents $N$. Using Theorem 1 we can partition the set of all voting by quotas $V b Q$ into equivalence classes in such a way that each equivalence class contains voting by quotas that are all equally manipulable. Denote the (quotient) set of those equivalence classes by $V b Q / \approx$. Furthermore, we can extend $\succsim$ on $V b Q$ to the set of equivalence classes $V b Q / \approx$ in a natural way. Denote this extension by [ $\succsim]$. In this subsection we will show that the pair $(V b Q / \approx,[\succsim])$ is a complete upper semilattice; namely, any nonempty subset of equivalence classes in $V b Q / \approx$ has a supremum according to [ $[$. Formally, given $\mathcal{W} \in V b Q$, denote by $[\mathcal{W}]$ the equivalence class of $\mathcal{W}$ with respect to $\approx$; i.e.,

$$
[\mathcal{W}]=\{\mathcal{V} \in V b Q \mid \mathcal{W} \approx \mathcal{V}\}
$$

By Corollary 1, $[\mathcal{W}]$ can be identified with the pair $\left(A_{1}^{\mathcal{W}}, A_{n}^{\mathcal{W}}\right)$. Denote by $V b Q / \approx$ the set of all equivalence classes induced by $\approx$ on $V b Q$ and consider the binary relation $[\succsim]$ on $V b Q / \approx$ defined as follows. For any pair $[\mathcal{W}],[\mathcal{V}] \in V b Q / \approx$, set $[\mathcal{V}][\succsim][\mathcal{W}]$ if and only if $\mathcal{V} \succsim \mathcal{W}$. 
Since $\succsim$ is a preorder on $V b Q$, it follows that $[\succsim]$ is a partial order on $V b Q / \approx$. Furthermore, by Corollary 1 ,

$$
[\mathcal{V}][\succsim][\mathcal{W}] \text { if and only if } A_{1}^{\mathcal{V}} \subset A_{1}^{\mathcal{W}} \text { and } A_{n}^{\mathcal{V}} \subset A_{n}^{\mathcal{W}}
$$

We can now state and prove the main result of this subsection.

Proposition 3 Assume $n \geq 3$. Then, the pair $(V b Q / \approx,[\succsim])$ is a complete upper semilattice.

Proof Let $\{\emptyset\} \neq Z \subseteq V b Q / \approx$. Consider any voting by quotas $\mathcal{V}: \mathcal{P}^{n} \rightarrow 2^{K}$ such that

$$
A_{1}^{\mathcal{V}}=\cap_{[\mathcal{W}] \in Z} A_{1}^{\mathcal{W}}, A_{n}^{\mathcal{V}}=\cap_{[\mathcal{W}] \in Z} A_{n}^{\mathcal{W}}, \text { and }
$$

for all $x \notin A_{1}^{\mathcal{V}} \cup A_{n}^{\mathcal{V}}$ (if any) set $q_{x}^{\mathcal{V}}$ equal to any integer other than 1 and $n$ (which exists because $n \geq 3$ ). Since $A_{1}^{\mathcal{W}} \cap A_{n}^{\mathcal{W}}=\{\emptyset\}$ for all $[\mathcal{W}] \in Z, \mathcal{V}$ is well defined as a voting by quotas. By Corollary 1 and the definition of $[\mathcal{V}], \sup _{[\succsim]} Z=[\mathcal{V}]$. Thus, $(V b Q / \approx,[\succsim])$ is a complete upper semilattice.

Example 2 and Figure 5 below show, for any $n \geq 3$, the complete upper semilattice structure of $(V b Q / \approx,[\succsim])$ when $|K|=3$.

Example 2 Assume $n \geq 3$ and let $K=\{x, y, z\}$ be the set of objects. Given $[\mathcal{W}] \in V b Q / \approx$ we identify the equivalence class $[\mathcal{W}]$ by the pair $\left(A_{1}^{\mathcal{W}}, A_{n}^{\mathcal{W}}\right)$ and furthermore we denote $\{x\}$ by $x,\{x, y\}$ by $x y$ and $\{x, y, z\}$ by $x y z$, and similarly for $\{y\},\{z\},\{x, z\}$, and $\{y, z\}$ (and $\{\emptyset\}$ by $\emptyset)$. By Proposition 3, the set of equivalence classes of voting by quotas is a complete upper semilattice. Figure 5 represents this partial order where if two pairs $\left(A_{1}^{\mathcal{W}}, A_{n}^{\mathcal{W}}\right)$ and $\left(A_{1}^{\mathcal{V}}, A_{n}^{\mathcal{V}}\right)$ are directly connected, the one above is more manipulable than the one below and moreover, all connections that can be obtained by transitivity are omitted. 


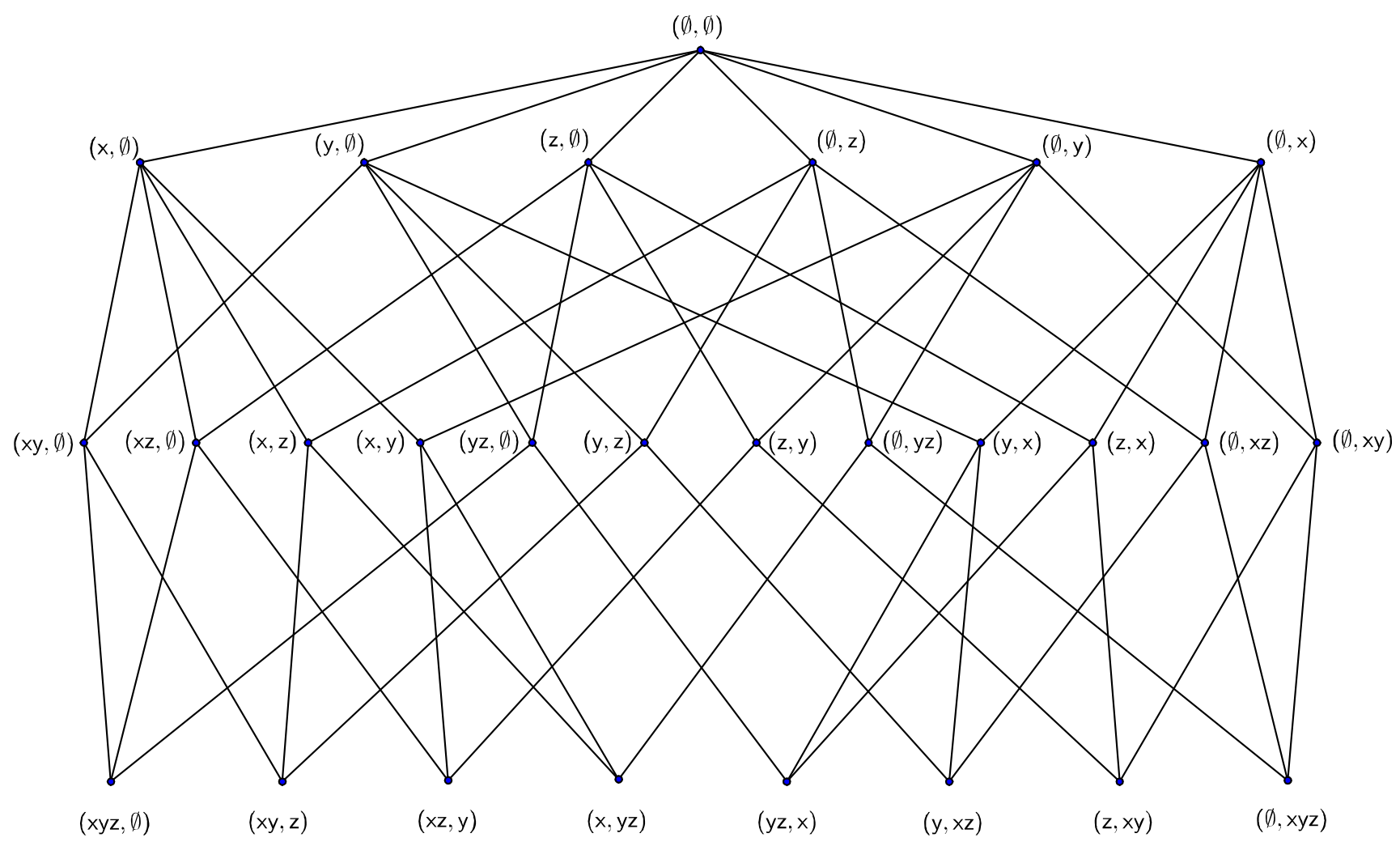

Figure 5

\section{Comparing all Voting by Committees}

In this section we state and prove the main result of the paper which identifies a set of conditions under which two voting by committees (with or without dummy agents) are comparable according to their manipulability. But before doing so we state a remark and give a definition.

Remark 5 Let $\mathcal{W}: \mathcal{P}^{n} \rightarrow 2^{K}$ be a voting by committees and let $x \in K$ and $i \in N$ be such that $x \in D u_{i}^{\mathcal{W}}$. Then, $x \in \overline{V e_{i}^{\mathcal{W}}}$ and $x \in \overline{D e_{i}^{\mathcal{W}}}$.

We say that a voting by committees $\mathcal{W}: \mathcal{P}^{n} \rightarrow 2^{K}$ is dictatorial if there exists $i \in N$ such that for all $x \in K, \mathcal{W}_{x}^{m}=\{\{i\}\}$. Obviously, all dictatorial voting by committees are strategy-proof in any domain and hence, they are less manipulable than any other voting by committees. Thus, it is not necessary to include them in the main result of the paper that we state below as Theorem 2 . 
Theorem 2 Let $\mathcal{V}: \mathcal{P}^{n} \rightarrow 2^{K}$ and $\mathcal{W}: \mathcal{P}^{n} \rightarrow 2^{K}$ be two non dictatorial voting by committees. Then, $\mathcal{V} \succsim \mathcal{W}$ if and only if for all $i \in N$,

$$
\begin{gathered}
D e_{i}^{\mathcal{V}} \subset D e_{i}^{\mathcal{W}} \text { and } V e_{i}^{\mathcal{V}} \subset V e_{i}^{\mathcal{W}} \text { and } D u_{i}^{\mathcal{V}} \subset D u_{i}^{\mathcal{W}} \text { or } \\
\left(D e_{i}^{\mathcal{V}} \backslash D e_{i}^{\mathcal{W}}\right) \cup\left(V e_{i}^{\mathcal{V}} \backslash V e_{i}^{\mathcal{W}}\right)=\{x\}=\overline{D u_{i}^{\mathcal{W}}} \text { or } \\
\overline{D e_{i}^{\mathcal{W}} \cap V e_{i}^{\mathcal{W}}}=\{x\}=D u_{i}^{\mathcal{V}} \backslash D u_{i}^{\mathcal{W}} .
\end{gathered}
$$

Before proving Theorem 2 we exhibit in Example 3 below two voting by committees that illustrate conditions (A), (B) and (C) above.

Example 3 Let $N=\{1,2,3\}$ be the set of agents and $K=\{x, y, z\}$ the set of objects. Consider two voting by committees $\mathcal{V}$ and $\mathcal{W}$ defined by the following (minimal) committees:

$$
\begin{array}{ll}
\mathcal{V}_{x}^{m}=\{\{1,2\},\{3\}\} & \mathcal{W}_{x}^{m}=\{\{3\}\} \\
\mathcal{V}_{y}^{m}=\{\{1\},\{2\}\} & \mathcal{W}_{y}^{m}=\{\{1\},\{2,3\}\} \\
\mathcal{V}_{z}^{m}=\{\{3\}\} & \mathcal{W}_{z}^{m}=\{\{3\}\} .
\end{array}
$$

We consider the decisive, vetoer and dummy sets of objects for each of the three agents separately.

For agent $1, D e_{1}^{\mathcal{V}}=D e_{1}^{\mathcal{W}}=\{y\}, V e_{1}^{\mathcal{V}}=V e_{1}^{\mathcal{W}}=\{\emptyset\}, D u_{1}^{\mathcal{V}}=\{z\}$ and $D u_{1}^{\mathcal{W}}=\{x, z\}$. Thus,

$$
D e_{1}^{\mathcal{V}}=D e_{1}^{\mathcal{W}} \text { and } V e_{1}^{\mathcal{V}}=V e_{1}^{\mathcal{W}} \text { and } D u_{1}^{\mathcal{V}} \subset D u_{1}^{\mathcal{W}}
$$

which means that condition (A) holds for agent 1. Observe that conditions (B) and (C) do not hold for agent 1 since $\overline{D u_{1}^{\mathcal{V}}}=\{y\}$ and $\left(D e_{1}^{\mathcal{V}} \backslash D e_{1}^{\mathcal{W}}\right) \cup\left(V e_{1}^{\mathcal{V}} \backslash V e_{1}^{\mathcal{W}}\right)=\{\emptyset\}$, and $\overline{D e_{1}^{\mathcal{W}} \cap V e_{1}^{\mathcal{W}}}=K$, respectively.

For agent 2, De $e_{2}^{\mathcal{V}}=\{y\}, D e_{2}^{\mathcal{W}}=\{\emptyset\}, V e_{2}^{\mathcal{V}}=V e_{2}^{\mathcal{W}}=\{\emptyset\}, D u_{2}^{\mathcal{V}}=\{z\}$ and $D u_{2}^{\mathcal{W}}=\{x, z\}$. Hence, $D e_{2}^{\mathcal{V}} \backslash D e_{2}^{\mathcal{W}}=\{y\}, V e_{2}^{\mathcal{V}} \backslash V e_{2}^{\mathcal{W}}=\{\emptyset\}$ and $\overline{D u_{2}^{\mathcal{V}}}=\{y\}$. Thus,

$$
\left(D e_{2}^{\mathcal{V}} \backslash D e_{2}^{\mathcal{W}}\right) \cup\left(V e_{2}^{\mathcal{V}} \backslash V e_{2}^{\mathcal{W}}\right)=\{y\}=\overline{D u_{2}^{\mathcal{W}}}
$$

which means that condition (B) holds for agent 2. Observe that conditions $(\mathrm{A})$ and $(\mathrm{C})$ do not hold for agent 2 since $D e_{2}^{\mathcal{V}} \nsubseteq D e_{2}^{\mathcal{W}}$, and $\overline{D e_{2}^{\mathcal{W}} \cap V e_{2}^{\mathcal{W}}}=K$, respectively.

For agent $3, D e_{3}^{\mathcal{V}}=D e_{3}^{\mathcal{W}}=\{x, z\}, V e_{3}^{\mathcal{V}}=\{z\}, V e_{3}^{\mathcal{W}}=\{x, z\}, D u_{3}^{\mathcal{V}}=\{y\}$ and $D u_{3}^{\mathcal{W}}=$ $\{\emptyset\}$. Hence, $D e_{3}^{\mathcal{W}} \cap V e_{3}^{\mathcal{W}}=\{x, z\}$ and $D u_{3}^{\mathcal{V}} \backslash D u_{3}^{\mathcal{W}}=\{y\}$. Thus,

$$
\overline{D e_{3}^{\mathcal{W}} \cap V e_{3}^{\mathcal{W}}}=\{y\}=D u_{3}^{\mathcal{V}} \backslash D u_{3}^{\mathcal{W}},
$$

which means that condition $(\mathrm{C})$ holds for agent 3. Observe that conditions $(\mathrm{A})$ and $(\mathrm{B})$ do not hold for agent 3 since $D u_{3}^{\mathcal{V}} \nsubseteq D u_{3}^{\mathcal{W}}$, and $\overline{D u_{3}^{\mathcal{W}}}=K$, respectively.

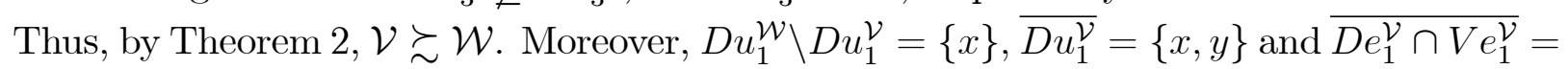
$\{x, y, z\}$. Hence, by Theorem $2, \mathcal{W} \succsim \mathcal{V}$ does not hold. Thus, $\mathcal{V} \succ \mathcal{W}$. 
We next exhibit a preference $P_{1}$ such that $P_{1} \in \mathcal{M}_{1}^{\mathcal{V}}$ but $P_{1} \notin \mathcal{M}_{1}^{\mathcal{V}}$. Let $P_{1}$ be the following preference:

$$
\{y\} P_{1}\{x, y, z\} P_{1}\{y, z\} P_{1}\{y, x\} P_{1}\{\emptyset\} P_{1}\{x\} P_{1}\{z\} P_{1}\{x, z\} .
$$

Let $\left(P_{1}^{\prime}, P_{2}^{\prime}, P_{3}^{\prime}\right) \in \mathcal{P}^{3}$ be any preference profile with the property that $t\left(P_{1}^{\prime}\right)=\{x, y\}, t\left(P_{2}^{\prime}\right)=$ $\{x\}$ and $t\left(P_{3}^{\prime}\right)=\{z\}$. Then,

$$
\mathcal{V}\left(P_{1}^{\prime}, P_{2}^{\prime}, P_{3}^{\prime}\right)=\{x, y, z\} P_{1}\{y, z\}=\mathcal{V}\left(P_{1}, P_{2}^{\prime}, P_{3}^{\prime}\right)
$$

and hence, $P_{1} \in \mathcal{M}_{1}^{\mathcal{V}}$. In the other hand, consider any arbitrary preference profile $\left(P_{1}^{*}, P_{2}, P_{3}\right) \in$ $\mathcal{P}^{3}$. Then, for some (potentially empty subset) $A \subset K \backslash\{y\}$,

$$
\mathcal{W}\left(P_{1}, P_{2}, P_{3}\right)=\{y\} \cup A \text { and } A \subset \mathcal{W}\left(P_{1}^{*}, P_{2}, P_{3}\right) \subset\{y\} \cup A
$$

Hence, by the definition of $P_{1}$,

$$
\mathcal{W}\left(P_{1}, P_{2}, P_{3}\right) R_{1} \mathcal{W}\left(P_{1}^{*}, P_{2}, P_{3}\right)
$$

Thus, $P_{1} \notin \mathcal{M}_{1}^{\mathcal{W}}$.

Proof of Theorem $2 \Leftarrow$ ) To prove sufficiency, fix $i \in N$ and assume that $P_{i} \in \mathcal{M}_{i}^{\mathcal{W}}$. We want to show that $P_{i} \in \mathcal{M}_{i}^{\mathcal{V}}$. By Lemma 4, there exist $A \in o^{\mathcal{W}}\left(P_{i}\right), T_{1} \subset t\left(P_{i}\right) \cap A \cap \overline{D u_{i}^{\mathcal{W}}}$ and $T_{2} \subset \overline{t\left(P_{i}\right)} \cap \bar{A} \cap \overline{D u_{i}^{\mathcal{W}}}$ such that

$$
\left(A \backslash T_{1}\right) \cup T_{2} P_{i} A
$$

We distinguish among three cases, depending on whether (A), (B) or (C) holds.

Case 1: Suppose that (A) holds; namely, $D e_{i}^{\mathcal{V}} \subset D e_{i}^{\mathcal{W}}, V e_{i}^{\mathcal{V}} \subset V e_{i}^{\mathcal{W}}$ and $D u_{i}^{\mathcal{V}} \subset D u_{i}^{\mathcal{W}}$. Hence,

$$
T_{1} \subset t\left(P_{i}\right) \cap A \cap \overline{D u_{i}^{\mathcal{V}}} \text { and } T_{2} \subset \overline{t\left(P_{i}\right)} \cap \bar{A} \cap \overline{D u_{i}^{\mathcal{V}}} .
$$

By Lemma 2, $A \in o^{\mathcal{V}}\left(P_{i}\right)$. By (14), (15) and Lemma 4, $P_{i} \in \mathcal{M}_{i}^{\mathcal{V}}$.

Case 2: Suppose that (B) holds; namely, $\left(D e_{i}^{\mathcal{V}} \backslash D e_{i}^{\mathcal{W}}\right) \cup\left(V e_{i}^{\mathcal{V}} \backslash V e_{i}^{\mathcal{W}}\right)=\{x\}=\overline{D u_{i}^{\mathcal{W}}}$. Since $x \in\left(D e_{i}^{\mathcal{V}} \backslash D e_{i}^{\mathcal{W}}\right) \cup\left(V e_{i}^{\mathcal{V}} \backslash V e_{i}^{\mathcal{W}}\right)$, either $x \in D e_{i}^{\mathcal{V}}$ or $x \in V e_{i}^{\mathcal{V}}$. By Remark $5, x \in \overline{D u_{i}^{\mathcal{V}}}$. Hence,

$$
T_{1} \subset t\left(P_{i}\right) \cap A \cap \overline{D u_{i}^{\mathcal{V}}} \text { and } T_{2} \subset \overline{t\left(P_{i}\right)} \cap \bar{A} \cap \overline{D u_{i}^{\mathcal{V}}}
$$

By (14), (16) and Lemma 4, it is sufficient to show that $A \in o^{\mathcal{V}}\left(P_{i}\right)$; or equivalently, by Lemma 2, that $t\left(P_{i}\right) \cap D e_{i}^{\mathcal{V}} \subset A$ and $A \subset t\left(P_{i}\right) \cup \overline{V e_{i}^{\mathcal{V}}}$. We distinguish between two subcases. Case 2.1: Suppose $x \notin t\left(P_{i}\right)$. Then, since $D e_{i}^{\mathcal{V}} \backslash D e_{i}^{\mathcal{W}} \subset\{x\}, t\left(P_{i}\right) \cap D e_{i}^{\mathcal{V}} \subset t\left(P_{i}\right) \cap D e_{i}^{\mathcal{W}}$ holds. By assumption, $A \in o^{\mathcal{W}}\left(P_{i}\right)$. Hence, $t\left(P_{i}\right) \cap D e_{i}^{\mathcal{W}} \subset A$. Thus,

$$
t\left(P_{i}\right) \cap D e_{i}^{\mathcal{V}} \subset A .
$$


Since $x \notin t\left(P_{i}\right)$ and $\overline{D u_{i}^{\mathcal{W}}}=\{x\}$ hold, we have that $t\left(P_{i}\right) \cap \overline{D u_{i}^{\mathcal{W}}}=\{\emptyset\}$ and $\overline{t\left(P_{i}\right)} \cap \overline{D u_{i}^{\mathcal{W}}}=$ $\{x\}$. Moreover, since $T_{1} \subset t\left(P_{i}\right) \cap A \cap \overline{D u_{i}^{\mathcal{W}}}, T_{2} \subset \overline{t\left(P_{i}\right)} \cap \bar{A} \cap \overline{D u_{i}^{\mathcal{W}}}$ and (14) hold, we have that $T_{1}=\{\emptyset\}$ and $T_{2}=\{x\}$ hold as well. Since $T_{2} \subset \bar{A}, x \notin A$. Hence, since $A \in o^{\mathcal{W}}\left(P_{i}\right)$,

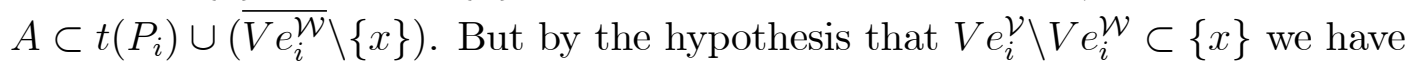

$$
A \subset t\left(P_{i}\right) \cup \overline{V e_{i}^{\mathcal{V}}}
$$

Case 2.2: Suppose $x \in t\left(P_{i}\right)$. Then, since $\overline{D u_{i}^{\mathcal{W}}}=\{x\}, t\left(P_{i}\right) \cap \overline{D u_{i}^{\mathcal{W}}}=\{x\}$ and $\overline{t\left(P_{i}\right)} \cap \overline{D u_{i}^{\mathcal{W}}}=$ $\{\emptyset\}$. Since $T_{1} \subset t\left(P_{i}\right) \cap A \cap \overline{D u_{i}^{\mathcal{W}}}, T_{2} \subset \overline{t\left(P_{i}\right)} \cap \bar{A} \cap \overline{D u_{i}^{\mathcal{W}}}$ and (14) hold, we have that $T_{1}=\{x\}$ and $T_{2}=\{\emptyset\}$ hold as well. Since $T_{1} \subset A, x \in A$. Hence, since $A \in o^{\mathcal{W}}\left(P_{i}\right)$, $\left(t\left(P_{i}\right) \cap D e_{i}^{\mathcal{W}}\right) \cup\{x\} \subset A$. But by the hypothesis that $D e_{i}^{\mathcal{V}} \backslash D e_{i}^{\mathcal{W}} \subset\{x\}$ we have

$$
t\left(P_{i}\right) \cap D e_{i}^{\mathcal{V}} \subset A .
$$

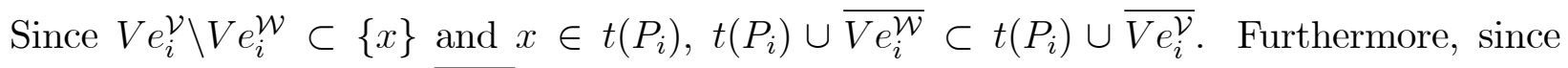
$A \in o^{\mathcal{W}}\left(P_{i}\right), A \subset t\left(P_{i}\right) \cup \overline{V e_{i}^{\mathcal{W}}}$. Thus,

$$
A \subset t\left(P_{i}\right) \cup \overline{V e_{i}^{\mathcal{V}}}
$$

Case 3: Suppose that (C) holds; namely, $\overline{D e_{i}^{\mathcal{W}} \cap V e_{i}^{\mathcal{W}}}=\{x\}=D u_{i}^{\mathcal{V}} \backslash D u_{i}^{\mathcal{W}}$. Since $x \in D u_{i}^{\mathcal{V}}$, by Remark $5, x \in \overline{V e_{i}^{\mathcal{V}}}$ and $x \in \overline{D e_{i}^{\mathcal{V}}}$. Hence,

$$
\begin{gathered}
D e_{i}^{\mathcal{V}} \subset K \backslash\{x\}=\overline{\overline{D e_{i}^{\mathcal{W}} \cap V e_{i}^{\mathcal{W}}}}=D e_{i}^{\mathcal{W}} \cap V e_{i}^{\mathcal{W}} \subset D e_{i}^{\mathcal{W}} \text { and } \\
\overline{V e_{i}^{\mathcal{W}}} \subset \overline{D e_{i}^{\mathcal{W}}} \cup \overline{V e_{i}^{\mathcal{W}}}=\overline{D e_{i}^{\mathcal{W}} \cap V e_{i}^{\mathcal{W}}}=\{x\} \subset \overline{V e_{i}^{\mathcal{V}}} .
\end{gathered}
$$

By Lemma 3, (21) and (22), $o^{\mathcal{W}}\left(P_{i}\right) \subset o^{\mathcal{V}}\left(P_{i}\right)$. Hence, and since $A \in o^{\mathcal{W}}\left(P_{i}\right), A \in o^{\mathcal{V}}\left(P_{i}\right)$. We want to prove that $P_{i} \in \mathcal{M}_{i}^{\mathcal{V}}$. By Lemma 4 and (14), it is sufficient to show that

$$
T_{1} \subset t\left(P_{i}\right) \cap A \cap \overline{D u_{i}^{\mathcal{V}}} \text { and } T_{2} \subset \overline{t\left(P_{i}\right)} \cap \bar{A} \cap \overline{D u_{i}^{\mathcal{V}}}
$$

hold. We distinguish between two subcases.

Case 3.1: Suppose $x \in t\left(P_{i}\right)$. As (C) holds, $\overline{V e_{i}^{\mathcal{W}}} \subset\{x\}$ and $K \backslash\{x\} \subset D e_{i}^{\mathcal{W}}$. Hence, by Lemma 2, o $o^{\mathcal{W}}\left(P_{i}\right) \subset\left\{t\left(P_{i}\right) \backslash\{x\}, t\left(P_{i}\right)\right\}$. By (14), $A=t\left(P_{i}\right) \backslash\{x\}$. Then, since $x \notin A$ and $x \in t\left(P_{i}\right), x \notin T_{1}$ and $x \notin T_{2}$ hold, respectively. Hence, and since $T_{1} \subset t\left(P_{i}\right) \cap A \cap \overline{D u_{i}^{\mathcal{W}}}$, $T_{2} \subset \overline{t\left(P_{i}\right)} \cap \bar{A} \cap \overline{D u_{i}^{\mathcal{W}}}$ and $D u_{i}^{\mathcal{V}} \backslash D u_{i}^{\mathcal{W}}=\{x\}$ hold, we have that $T_{1} \subset t\left(P_{i}\right) \cap A \cap \overline{D u_{i}^{\mathcal{V}}}$ and $T_{2} \subset \overline{t\left(P_{i}\right)} \cap \bar{A} \cap \overline{D u_{i}^{\mathcal{V}}}$ hold as well.

Case 3.2: Suppose $x \notin t\left(P_{i}\right)$. As $(\mathrm{C})$ holds, $\overline{V e_{i}^{\mathcal{W}}} \subset\{x\}$ and $K \backslash\{x\} \subset D e_{i}^{\mathcal{W}}$. Hence, by Lemma 2, o $o^{\mathcal{W}}\left(P_{i}\right) \subset\left\{t\left(P_{i}\right), t\left(P_{i}\right) \cup\{x\}\right\}$. By (14), $A=t\left(P_{i}\right) \cup\{x\}$. Then, since $x \notin t\left(P_{i}\right)$ and $x \in A, x \notin T_{1}$ and $x \notin T_{2}$ hold, respectively. Hence, and since $T_{1} \subset t\left(P_{i}\right) \cap A \cap \overline{D u_{i}^{\mathcal{W}}}$, $T_{2} \subset \overline{t\left(P_{i}\right)} \cap \bar{A} \cap \overline{D u_{i}^{\mathcal{V}}}$ and $D u_{i}^{\mathcal{V}} \backslash D u_{i}^{\mathcal{W}}=\{x\}$ hold, we have that $T_{1} \subset t\left(P_{i}\right) \cap A \cap \overline{D u_{i}^{\mathcal{V}}}$ and $T_{2} \subset \overline{t\left(P_{i}\right)} \cap \bar{A} \cap \overline{D u_{i}^{\mathcal{V}}}$ hold as well. 
$\Rightarrow)$ To prove necessity, assume $\mathcal{V}$ and $\mathcal{W}$ are non dictatorial voting by committees and $\mathcal{V} \succsim \mathcal{W}$; i.e.,

$$
\mathcal{M}_{i}^{\mathcal{W}} \subset \mathcal{M}_{i}^{\mathcal{V}} \text { for all } i \in N
$$

Fix $i \in N$ and assume that (A) and (B) do not hold. We will show that (C) holds; i.e.,

$$
\overline{D e_{i}^{\mathcal{W}} \cap V e_{i}^{\mathcal{W}}}=\{x\}=D u_{i}^{\mathcal{V}} \backslash D u_{i}^{\mathcal{W}} .
$$

Claim 1 If $\left|D u_{i}^{\mathcal{V}} \backslash D u_{i}^{\mathcal{W}}\right| \geq 2$ and $\mathcal{V} \succsim \mathcal{W}$, then $i$ is a dictator in $\mathcal{W}$.

Proof of Claim 1 Assume $x \in D u_{i}^{\mathcal{V}} \backslash D u_{i}^{\mathcal{V}}$ and suppose that there exists $y \in K$ such that $y \notin D e_{i}^{\mathcal{W}}$. Since $\left|D u_{i}^{\mathcal{V}} \backslash D u_{i}^{\mathcal{W}}\right| \geq 2$ we can assume without loss of generality that $y \neq x$. Let $P_{i}^{\prime} \in \mathcal{P}$ be any preference such that $t\left(P_{i}^{\prime}\right)=\{x, y\}$ and satisfying in addition the following properties:

(i) $\{x, y\} P_{i}^{\prime}\{y\} P_{i}^{\prime}\{\emptyset\} P_{i}^{\prime}\{x\} P_{i}^{\prime} A$,

for all $A \notin\{\{x, y\},\{y\},\{\emptyset\},\{x\}\}$,

(ii) $A R_{i}^{\prime} B$ if $B \cap t\left(P_{i}^{\prime}\right) \subset A \cap t\left(P_{i}^{\prime}\right)$ and $A \cap \overline{t\left(P_{i}^{\prime}\right)} \subset B \cap \overline{t\left(P_{i}^{\prime}\right)}$,

for all $A, B \notin\{\{x, y\},\{y\},\{\emptyset\},\{x\}\}$.

The preference $P_{i}^{\prime}$ can be seen as having two separate blocks. The first one orders the subsets $\{x, y\},\{y\},\{\emptyset\},\{x\}$ in a nonseparable way. All other subsets are dispreferred to each of these four subsets but, among those that are different to these four, any set $A$ that can be obtained from $B$ by adding objects in $t\left(P_{i}^{\prime}\right)$ and deleting objects in $\overline{t\left(P_{i}^{\prime}\right)}$ is preferred to $B$. Moreover, any pair of subsets of objects that are unrestricted by conditions (i) and (ii) can be ordered arbitrarily by $P_{i}^{\prime}$.

Since $y \notin D e_{i}^{\mathcal{W}},\{x\} \in o^{\mathcal{W}}\left(P_{i}^{\prime}\right)$. Set $A=\{x\}, T_{2}=\{\emptyset\}$ and $T_{1}=\{x\}$. Observe that $A \in$ $o^{\mathcal{W}}\left(P_{i}^{\prime}\right)$ and, by (i) in the definition of $P_{i}^{\prime},\left(A \backslash T_{1}\right) \cup T_{2}=\{\emptyset\} P_{i}^{\prime}\{x\}=A$. Hence, by Lemma 4, $P_{i}^{\prime} \in \mathcal{M}_{i}^{\mathcal{W}}$. Take now any $A \in o^{\mathcal{V}}\left(P_{i}^{\prime}\right)$. Let $T_{1} \subset t\left(P_{i}^{\prime}\right) \cap A \cap \overline{D u_{i}^{\mathcal{V}}}$ and $T_{2} \subset \overline{t\left(P_{i}^{\prime}\right)} \cap \bar{A} \cap \overline{D u_{i}^{\mathcal{V}}}$ be arbitrary. We can assume that either $T_{1} \neq\{\emptyset\}$ or $T_{2} \neq\{\emptyset\}$; otherwise, $A=\left(A \backslash T_{1}\right) \cup T_{2}$ and $A I_{i}^{\prime}\left(A \backslash T_{1}\right) \cup T_{2}$ trivially. Since $x \in D u_{i}^{\mathcal{V}}$ and $t\left(P_{i}^{\prime}\right)=\{x, y\}, T_{1} \subset\{y\}$ and $x, y \notin T_{2}$. We distinguish between two cases.

Case 1.a: If $\left(A \backslash T_{1}\right) \cup T_{2} \notin\{\{x, y\},\{y\},\{\emptyset\},\{x\}\}$ then, by (i) and (ii) in the definition of $P_{i}^{\prime}, A R_{i}^{\prime}\left(A \backslash T_{1}\right) \cup T_{2}$.

Case 2.a: If $\left(A \backslash T_{1}\right) \cup T_{2} \in\{\{x, y\},\{y\},\{\emptyset\},\{x\}\}$ then $T_{2}=\{\emptyset\}$ and $T_{1}=\{y\}$. Hence, $y \in A$ and $\left(A \backslash T_{1}\right) \cup T_{2}=A \backslash\{y\}$. Since $\left(A \backslash T_{1}\right) \cup T_{2} \in\{\{x, y\},\{y\},\{\emptyset\},\{x\}\}$ and $\left(A \backslash T_{1}\right) \cup T_{2}=$ $A \backslash\{y\}$, either $A=\{y\}$ and $\left(A \backslash T_{1}\right) \cup T_{2}=\{\emptyset\}$ or else $A=\{x, y\}$ and $\left(A \backslash T_{1}\right) \cup T_{2}=\{x\}$. But then, and according to (i) in the definition of $P_{i}^{\prime}, A P_{i}^{\prime}\left(A \backslash T_{1}\right) \cup T_{2}$ must hold.

Hence, by Lemma $4, P_{i}^{\prime} \notin \mathcal{M}_{i}^{\mathcal{V}}$ in both cases. Thus, $P_{i}^{\prime} \in \mathcal{M}_{i}^{\mathcal{W}} \backslash \mathcal{M}_{i}^{\mathcal{V}}$, which contradicts that $\mathcal{V} \succsim \mathcal{W}$. Therefore, we cannot suppose that there exists $y \in K$ such that $y \notin D e_{i}^{\mathcal{W}}$. Thus,

$$
D e_{i}^{\mathcal{W}}=K
$$


Now, suppose that there exists $y \in K$ such that $y \in \overline{V e_{i}^{\mathcal{W}}}$. Since $x \in D u_{i}^{\mathcal{V}} \backslash D u_{i}^{\mathcal{W}}$ and $\left|D u_{i}^{\mathcal{V}} \backslash D u_{i}^{\mathcal{W}}\right| \geq 2$ we can assume without loss of generality that $y \neq x$. Let $P_{i}^{\prime \prime} \in \mathcal{P}$ be any preference such that $t\left(P_{i}^{\prime \prime}\right)=\{x\}$ and satisfying in addition the following properties:

(i) $\{x\} P_{i}^{\prime \prime}\{\emptyset\} P_{i}^{\prime \prime}\{y\} P_{i}^{\prime \prime}\{x, y\} P_{i}^{\prime \prime} A$,

for all $A \notin\{\{x\},\{\emptyset\},\{y\},\{x, y\}\}$,

(ii) $A R_{i}^{\prime \prime} B$ if $B \cap t\left(P_{i}^{\prime \prime}\right) \subset A \cap t\left(P_{i}^{\prime \prime}\right)$ and $A \cap \overline{t\left(P_{i}^{\prime \prime}\right)} \subset B \cap \overline{t\left(P_{i}^{\prime \prime}\right)}$,

for all $A, B \notin\{\{x\},\{\emptyset\},\{y\},\{x, y\}\}$.

The preference $P_{i}^{\prime \prime}$ can also be seen as having two separate blocks. The first one orders the subsets $\{x\},\{\emptyset\},\{y\},\{x, y\}$ in a nonseparable way. All other subsets are dispreferred to each of these four subsets but, among those that are different to these four, any set $A$ that can be obtained from $B$ by adding objects in $t\left(P_{i}^{\prime \prime}\right)$ and deleting objects in $\overline{t\left(P_{i}^{\prime \prime}\right)}$ is preferred to $B$. Moreover, any pair of subsets of objects that are unrestricted by conditions (i) and (ii) can be ordered arbitrarily by $P_{i}^{\prime \prime}$.

Since $y \in \overline{V e_{i}^{\mathcal{W}}},\{x, y\} \in o^{\mathcal{W}}\left(P_{i}^{\prime \prime}\right)$. Set $A=\{x, y\}, T_{1}=\{x\}$ and $T_{2}=\{\emptyset\}$. Observe that $A \in o^{\mathcal{W}}\left(P_{i}^{\prime \prime}\right)$ and, by (i) in the definition of $P_{i}^{\prime \prime},\left(A \backslash T_{1}\right) \cup T_{2}=\{y\} P_{i}^{\prime \prime}\{x, y\}=A$. Hence, by Lemma $4, P_{i}^{\prime \prime} \in \mathcal{M}_{i}^{\mathcal{W}}$. Take now any $A \in o^{\mathcal{V}}\left(P_{i}^{\prime \prime}\right)$. Let $T_{1} \subset t\left(P_{i}^{\prime \prime}\right) \cap A \cap \overline{D u_{i}^{\mathcal{V}}}$ and $T_{2} \subset \overline{t\left(P_{i}^{\prime \prime}\right)} \cap \bar{A} \cap \overline{D u_{i}^{\mathcal{V}}}$ be arbitrary. We can assume that either $T_{1} \neq\{\emptyset\}$ or $T_{2} \neq\{\emptyset\}$; otherwise, $A=\left(A \backslash T_{1}\right) \cup T_{2}$ and $A I_{i}^{\prime \prime}\left(A \backslash T_{1}\right) \cup T_{2}$ trivially. Since $x \in D u_{i}^{\mathcal{V}}$ and $t\left(P_{i}^{\prime \prime}\right)=\{x\}$, $T_{1}=\{\emptyset\}$ and $x \notin T_{2}$. We distinguish between two cases.

Case 1.b: If $\left(A \backslash T_{1}\right) \cup T_{2} \notin\{\{x\},\{\emptyset\},\{y\},\{x, y\}\}$ then, by (i) and (ii) in the definition of $P_{i}^{\prime \prime}, A R_{i}^{\prime \prime}\left(A \backslash T_{1}\right) \cup T_{2}$.

Case 2.b: If $\left(A \backslash T_{1}\right) \cup T_{2} \in\{\{x\},\{\emptyset\},\{y\},\{x, y\}\}$ then $T_{2} \subset\{x, y\}$. Since $T_{1}=\{\emptyset\}, x \notin T_{2}$ and $T_{2} \neq\{\emptyset\}, T_{2}=\{y\}$ and $y \notin A$. Then, either $A=\{\emptyset\}$ and $\left(A \backslash T_{1}\right) \cup T_{2}=\{y\}$ or else $A=\{x\}$ and $\left(A \backslash T_{1}\right) \cup T_{2}=\{x, y\}$. But then, and according to (i) in the definition of $P_{i}^{\prime \prime}$, $A P_{i}^{\prime \prime}\left(A \backslash T_{1}\right) \cup T_{2}$ must hold.

Hence, by Lemma $4, P_{i}^{\prime \prime} \notin \mathcal{M}_{i}^{\mathcal{V}}$ in both cases. Thus, $P_{i}^{\prime \prime} \in \mathcal{M}_{i}^{\mathcal{W}} \backslash \mathcal{M}_{i}^{\mathcal{V}}$, which contradicts that $\mathcal{V} \succsim \mathcal{W}$. Therefore, we can not suppose that there exists $y \in K$ such that $y \in \overline{V e_{i}^{\mathcal{W}}}$. Thus,

$$
\overline{V e_{i}^{\mathcal{W}}}=\{\emptyset\}
$$

By (25) and (26), $i$ is a dictator in $\mathcal{W}$. This finishes the proof of Claim 1.

By hypothesis, $\mathcal{W}$ is non dictatorial. By Claim 1,

$$
\left|D u_{i}^{\mathcal{V}} \backslash D u_{i}^{\mathcal{W}}\right| \leq 1
$$

We distinguish between two cases. 
Case I: Suppose that $D u_{i}^{\mathcal{V}} \backslash D u_{i}^{\mathcal{W}}=\{x\}$. We want to show that $\overline{D e_{i}^{\mathcal{W}} \cap V e_{i}^{\mathcal{W}}}=\{x\}$. As $x \in D u_{i}^{\mathcal{V}} \backslash D u_{i}^{\mathcal{W}}$, applying the same argument than the one used in the proof of Claim 1, we can obtain that $\overline{D e_{i}^{\mathcal{W}}} \subset\{x\}$ and $\overline{V e_{i}^{\mathcal{W}}} \subset\{x\}$. Therefore,

$$
\overline{D e_{i}^{\mathcal{W}} \cap V e_{i}^{\mathcal{W}}}=\overline{D e_{i}^{\mathcal{W}}} \cup \overline{V e_{i}^{\mathcal{W}}} \subset\{x\}
$$

Furthermore, since $i$ is not a dictator in $\mathcal{W}, \overline{D e_{i}^{\mathcal{W}}} \neq\{\emptyset\}$ or $\overline{V e_{i}^{\mathcal{W}}} \neq\{\emptyset\}$. Hence, by (27),

$$
\overline{D e_{i}^{\mathcal{W}} \cap V e_{i}^{\mathcal{W}}}=\overline{D e_{i}^{\mathcal{W}}} \cup \overline{V e_{i}^{\mathcal{W}}}=\{x\}=D u_{i}^{\mathcal{V}} \backslash D u_{i}^{\mathcal{W}}
$$

Case II: Suppose that $D u_{i}^{\mathcal{V}} \backslash D u_{i}^{\mathcal{V}}=\{\emptyset\}$. We will obtain a contradiction. Since (A) does not hold there exists $x \in K$ such that $x \in\left(D e_{i}^{\mathcal{V}} \backslash D e_{i}^{\mathcal{W}}\right) \cup\left(V e_{i}^{\mathcal{V}} \backslash V e_{i}^{\mathcal{W}}\right)$.

Claim $2 \quad \overline{D u_{i}^{\mathcal{W}}}=\{x\}$.

Proof of Claim 2 We distinguish between two cases.

Case 1: Assume $x \in D e_{i}^{\mathcal{V}} \backslash D e_{i}^{\mathcal{W}}$ and there exists $y \neq x$ such that $y \in \overline{D u_{i}^{\mathcal{V}}}$. Let $P_{i}^{\prime \prime \prime} \in \mathcal{P}$ be any preference such that $t\left(P_{i}^{\prime \prime \prime}\right)=\{x, y\}$ and satisfying in addition the following properties:

(i) $\{x, y\} P_{i}^{\prime \prime \prime}\{x\} P_{i}^{\prime \prime \prime}\{\emptyset\} P_{i}^{\prime \prime \prime}\{y\} P_{i}^{\prime \prime \prime} A$

for all $A \notin\{\{x, y\},\{y\},\{\emptyset\},\{x\}\}$,

(ii) $A R_{i}^{\prime \prime \prime} B$ if $B \cap t\left(P_{i}^{\prime \prime \prime}\right) \subset A \cap t\left(P_{i}^{\prime \prime \prime}\right)$ and $A \cap \overline{t\left(P_{i}^{\prime \prime \prime}\right)} \subset B \cap \overline{t\left(P_{i}^{\prime \prime \prime}\right)}$, for all $A, B \notin\{\{x, y\},\{y\},\{\emptyset\},\{x\}\}$.

As previously the preference $P_{i}^{\prime \prime \prime}$ can also be seen as having two separate blocks (we omit the details).

Since $x \in D e_{i}^{\mathcal{V}} \backslash D e_{i}^{\mathcal{W}},\{y\} \in o^{\mathcal{W}}\left(P_{i}^{\prime \prime \prime}\right)$. Set $A=\{y\}, T_{2}=\{\emptyset\}$ and $T_{1}=\{y\}$. Observe that $A \in o^{\mathcal{W}}\left(P_{i}^{\prime \prime \prime}\right)$ and, by (i) in the definition of $P_{i}^{\prime \prime \prime},\left(A \backslash T_{1}\right) \cup T_{2}=\{\emptyset\} P_{i}^{\prime \prime \prime}\{y\}=A$. Hence, by Lemma $4, P_{i}^{\prime \prime \prime} \in \mathcal{M}_{i}^{\mathcal{W}}$. Take now any $A \in o^{\mathcal{V}}\left(P_{i}^{\prime \prime \prime}\right)$. Since $x \in D e_{i}^{\mathcal{V}} \backslash D e_{i}^{\mathcal{W}}$ and $x \in t\left(P_{i}^{\prime \prime \prime}\right)$, $x \in A$. Let $T_{1} \subset t\left(P_{i}^{\prime \prime \prime}\right) \cap A \cap \overline{D u_{i}^{\mathcal{V}}}$ and $T_{2} \subset \overline{t\left(P_{i}^{\prime \prime \prime}\right)} \cap \bar{A} \cap \overline{D u_{i}^{\mathcal{V}}}$ be arbitrary. We can assume that either $T_{1} \neq\{\emptyset\}$ or $T_{2} \neq\{\emptyset\}$; otherwise, $A=\left(A \backslash T_{1}\right) \cup T_{2}$ and $A I_{i}^{\prime \prime \prime}\left(A \backslash T_{1}\right) \cup T_{2}$ trivially. Since $t\left(P_{i}^{\prime \prime \prime}\right)=\{x, y\}, T_{1} \subset\{x, y\}$ and $x, y \notin T_{2}$. Now we will consider two subcases.

Case 1.1: If $\left(A \backslash T_{1}\right) \cup T_{2} \notin\{\{x, y\},\{y\},\{\emptyset\},\{x\}\}$, then by (i) and (ii) in the definition of $P_{i}^{\prime \prime \prime}, A R_{i}^{\prime \prime \prime}\left(A \backslash T_{1}\right) \cup T_{2}$.

Case 1.2: If $\left(A \backslash T_{1}\right) \cup T_{2} \in\{\{x, y\},\{y\},\{\emptyset\},\{x\}\}$, then $T_{2}=\{\emptyset\}$; otherwise, if there exists $z \in T_{2} \backslash\{x, y\}$, then $z \in\left(A \backslash T_{1}\right) \cup T_{2}$ and therefore $\left(A \backslash T_{1}\right) \cup T_{2} \notin\{\{x, y\},\{y\},\{\emptyset\},\{x\}\}$. Hence, $T_{1} \neq\{\emptyset\}$ and $\left(A \backslash T_{1}\right) \cup T_{2}=A \backslash T_{1}$. We distinguish among three different subcases.

Case 1.2.1: $T_{1}=\{x\}$. Then, $x \in A$ and $\left(A \backslash T_{1}\right) \cup T_{2}=A \backslash\{x\}$. Since $\left(A \backslash T_{1}\right) \cup T_{2} \in$ $\{\{x, y\},\{y\},\{\emptyset\},\{x\}\}$ and $\left(A \backslash T_{1}\right) \cup T_{2}=A \backslash\{x\}$, either $\left(A \backslash T_{1}\right) \cup T_{2}=\{y\}$ and $A=\{x, y\}$ or else $\left(A \backslash T_{1}\right) \cup T_{2}=\{\emptyset\}$ and $A=\{x\}$ (since $x \in A$ ). But in both cases, by (i) in the definition of $P_{i}^{\prime \prime \prime}, A P_{i}^{\prime \prime \prime}\left(A \backslash T_{1}\right) \cup T_{2}$. 
Case 1.2.2: $T_{1}=\{y\}$. Then, $y \in A$ and $\left(A \backslash T_{1}\right) \cup T_{2}=A \backslash\{y\}$. Since $\left(A \backslash T_{1}\right) \cup T_{2} \in$ $\{\{x, y\},\{y\},\{\emptyset\},\{x\}\}$ and $\left(A \backslash T_{1}\right) \cup T_{2}=A \backslash\{y\},\left(A \backslash T_{1}\right) \cup T_{2}=\{x\}$ and $A=\{x, y\}$ (since $x \in A)$. But then, by (i) in the definition of $P_{i}^{\prime \prime \prime}, A P_{i}^{\prime \prime \prime}\left(A \backslash T_{1}\right) \cup T_{2}$.

Case 1.2.3: $T_{1}=\{x, y\}$. Then, $x, y \in A$ and $\left(A \backslash T_{1}\right) \cup T_{2}=A \backslash\{y, x\}$. Since $\left(A \backslash T_{1}\right) \cup T_{2} \in$ $\{\{x, y\},\{y\},\{\emptyset\},\{x\}\}$ and $\left(A \backslash T_{1}\right) \cup T_{2}=A \backslash\{y, x\},\left(A \backslash T_{1}\right) \cup T_{2}=\{\emptyset\}$ and $A=\{x, y\}$ (since $x, y \in A)$. But then, by (i) in the definition of $P_{i}^{\prime \prime \prime}, A P_{i}^{\prime \prime \prime}\left(A \backslash T_{1}\right) \cup T_{2}$.

Hence, by Lemma $4, P_{i}^{\prime \prime \prime} \notin \mathcal{M}_{i}^{\mathcal{V}}$. Thus, $P_{i}^{\prime \prime \prime} \in \mathcal{M}_{i}^{\mathcal{W}} \backslash \mathcal{M}_{i}^{\mathcal{V}}$ holds, which contradicts that $\mathcal{V} \succsim \mathcal{W}$

Case 2: Assume $x \in V e_{i}^{\mathcal{V}} \backslash V e_{i}^{\mathcal{W}}$ and there exists $y \neq x$ such that $y \in \overline{D u_{i}^{\mathcal{W}}}$. Let $P_{i}^{\prime \prime \prime \prime} \in P$ be any preference such that $t\left(P_{i}^{\prime \prime \prime \prime}\right)=\{y\}$ and satisfying in addition the following properties:

(i) $\{y\} P_{i}^{\prime \prime \prime \prime}\{\emptyset\} P_{i}^{\prime \prime \prime \prime}\{x\} P_{i}^{\prime \prime \prime \prime}\{x, y\} P_{i}^{\prime \prime \prime \prime} A$

for all $A \notin\{\{x, y\},\{y\},\{\emptyset\},\{x\}\}$,

(ii) $A R_{i}^{\prime \prime \prime \prime} B$ if $B \cap t\left(P_{i}^{\prime \prime \prime \prime}\right) \subset A \cap t\left(P_{i}^{\prime \prime \prime \prime}\right)$ and $A \cap \overline{t\left(P_{i}^{\prime \prime \prime \prime}\right)} \subset B \cap \overline{t\left(P_{i}^{\prime \prime \prime \prime}\right)}$,

for all $A, B \notin\{\{x, y\},\{y\},\{\emptyset\},\{x\}\}$.

As previously the non separable preference $P_{i}^{\prime \prime \prime \prime}$ can also be seen as having two separate blocks (we omit the details).

Since $x \in V e_{i}^{\mathcal{V}} \backslash V e_{i}^{\mathcal{W}},\{x, y\} \in o^{\mathcal{W}}\left(P_{i}^{\prime \prime \prime \prime}\right)$. Set $A=\{x, y\}, T_{1}=\{y\}$ and $T_{2}=\{\emptyset\}$. Observe that $A \in o^{\mathcal{W}}\left(P_{i}^{\prime \prime \prime \prime}\right)$ and, by (i) in the definition of $P_{i}^{\prime \prime \prime \prime},\left(A \backslash T_{1}\right) \cup T_{2}=\{x\} P_{i}^{\prime \prime \prime \prime}\{x, y\}=A$. Hence, by Lemma $4, P_{i}^{\prime \prime \prime \prime} \in \mathcal{M}_{i}^{\mathcal{W}}$. Take now any $A \in o^{\mathcal{V}}\left(P_{i}^{\prime \prime \prime \prime}\right)$. Since $x \in V e_{i}^{\mathcal{V}} \backslash V e_{i}^{\mathcal{W}}$ and $x \notin t\left(P_{i}^{\prime \prime \prime \prime}\right), x \notin A$. Let $T_{1} \subset t\left(P_{i}^{\prime \prime \prime \prime}\right) \cap A \cap \overline{D u_{i}^{\mathcal{V}}}$ and $T_{2} \subset \overline{t\left(P_{i}^{\prime \prime \prime \prime}\right)} \cap \bar{A} \cap \overline{D u_{i}^{\mathcal{V}}}$ be arbitrary. We can assume that either $T_{1} \neq\{\emptyset\}$ or $T_{2} \neq\{\emptyset\}$; otherwise, $A=\left(A \backslash T_{1}\right) \cup T_{2}$ and $A I_{i}^{\prime \prime \prime \prime}\left(A \backslash T_{1}\right) \cup T_{2}$ trivially. Since $t\left(P_{i}^{\prime \prime \prime \prime}\right)=\{y\}, T_{1} \subset\{y\}$ and $y \notin T_{2}$. We distinguish between two subcases.

Case 2.1: If $\left(A \backslash T_{1}\right) \cup T_{2} \notin\{\{x, y\},\{y\},\{\emptyset\},\{x\}\}$, then by (i) and (ii) in the definition of $P_{i}^{\prime \prime \prime \prime}, A R_{i}^{\prime \prime \prime \prime}\left(A \backslash T_{1}\right) \cup T_{2}$.

Case 2.2: If $\left(A \backslash T_{1}\right) \cup T_{2} \in\{\{x, y\},\{y\},\{\emptyset\},\{x\}\}$, then $T_{2} \subset\{x\}$. We distinguish among three different subcases.

Case 2.2.1: $T_{1}=\{\emptyset\}$ and $T_{2}=\{x\}$. Then, $\left(A \backslash T_{1}\right) \cup T_{2}=A \cup\{x\}$. Since $\left(A \backslash T_{1}\right) \cup T_{2} \in$ $\{\{x, y\},\{y\},\{\emptyset\},\{x\}\}$ and $\left(A \backslash T_{1}\right) \cup T_{2}=A \cup\{x\}$, either $\left(A \backslash T_{1}\right) \cup T_{2}=\{x, y\}$ and $A=\{y\}$ or else $\left(A \backslash T_{1}\right) \cup T_{2}=\{x\}$ and $A=\{\emptyset\}$ (since $x \notin A$ ). But then, by (i) in the definition of $P_{i}^{\prime \prime \prime \prime}, A P_{i}^{\prime \prime \prime \prime}\left(A \backslash T_{1}\right) \cup T_{2}$.

Case 2.2.2: $T_{1}=\{y\}$ and $T_{2}=\{\emptyset\}$. Then, $y \in A$ and $\left(A \backslash T_{1}\right) \cup T_{2}=A \backslash\{y\}$. Since $\left(A \backslash T_{1}\right) \cup T_{2} \in\{\{x, y\},\{y\},\{\emptyset\},\{x\}\}$ and $\left(A \backslash T_{1}\right) \cup T_{2}=A \backslash\{y\}$, either $\left(A \backslash T_{1}\right) \cup T_{2}=\{\emptyset\}$ and $A=\{y\}$ or else $\left(A \backslash T_{1}\right) \cup T_{2}=\{x\}$ and $A=\{y\}$ (since $x \notin A$ ). But then, by (i) in the definition of $P_{i}^{\prime \prime \prime \prime} A P_{i}^{\prime \prime \prime \prime}\left(A \backslash T_{1}\right) \cup T_{2}$. 
Case 2.2.3: $T_{1}=\{y\}$ and $T_{2}=\{x\}$. Then, $y \in A$ and $\left(A \backslash T_{1}\right) \cup T_{2}=A \backslash\{y\} \cup\{x\}$. Since $\left(A \backslash T_{1}\right) \cup T_{2} \in\{\{x, y\},\{y\},\{\emptyset\},\{x\}\}$ and $\left(A \backslash T_{1}\right) \cup T_{2}=A \backslash\{y\} \cup\{x\},\left(A \backslash T_{1}\right) \cup T_{2}=\{x\}$ and $A=\{y\}$ (since $x \notin A$ ). But then, by (i) in the definition of $P_{i}^{\prime \prime \prime \prime}, A P_{i}^{\prime \prime \prime \prime}\left(A \backslash T_{1}\right) \cup T_{2}$.

Hence, by Lemma $4, P_{i}^{\prime \prime \prime \prime} \notin \mathcal{M}_{i}^{\mathcal{V}}$. Thus, $P_{i}^{\prime \prime \prime \prime} \in \mathcal{M}_{i}^{\mathcal{W}} \backslash \mathcal{M}_{i}^{\mathcal{V}}$ holds, which contradicts that $\mathcal{V} \succsim \mathcal{W}$

Therefore, $\overline{D u_{i}^{\mathcal{W}}} \subset\{x\}$, Furthermore, and since we have assumed without loss of generality that $\overline{D u_{i}^{\mathcal{V}}} \neq\{\emptyset\}($ see $(3))$,

$$
\overline{D u_{i}^{\mathcal{W}}}=\{x\}
$$

This finishes the proof of Claim 2.

Finally, assume there exists $y \neq x$ such that $y \in\left(D e_{i}^{\mathcal{V}} \backslash D e_{i}^{\mathcal{W}}\right) \cup\left(V e_{i}^{\mathcal{V}} \backslash V e_{i}^{\mathcal{W}}\right)$. By Claim $2, \overline{D u_{i}^{\mathcal{W}}}=\{y\}$ which contradicts (28). Therefore,

$$
\left(D e_{i}^{\mathcal{V}} \backslash D e_{i}^{\mathcal{W}}\right) \cup\left(V e_{i}^{\mathcal{V}} \backslash V e_{i}^{\mathcal{W}}\right)=\{x\}=\overline{D u_{i}^{\mathcal{W}}}
$$

which contradicts that (B) does not hold. Therefore, Case II is not possible.

\section{Final Remark}

Before finishing the paper we want to relate our comparability criterion to two alternative criteria proposed by Pathak and Sönmez (2013). To present them in a more general setting let $X$ be a set of alternatives and now let $\mathcal{P}$ be the set of all strict preferences on $X$. Following Pathak and Sönmez (2013) we say that the profile $P \in \mathcal{P}^{n}$ is vulnerable under the social choice function $f: \mathcal{P}^{n} \rightarrow X$ if $f$ is manipulable by some agent at $P$; i.e., there exist $i \in N$ and $P_{i}^{\prime} \in \mathcal{P}$ such that $f\left(P_{i}^{\prime}, P_{-i}\right) P_{i} f\left(P_{i}, P_{-i}\right)$.

First, and following their definitions in Section 1, a social choice function $f: \mathcal{P}^{n} \rightarrow X$ is at least as manipulable as social choice function $g: \mathcal{P}^{n} \rightarrow X$ according to Pathak and Sönmez (referred to as at least as PS-manipulable as, and written as $f \succeq^{P S} g$ ) if any profile that is vulnerable under $g$ is also vulnerable under $f$; i.e.,

- if there exist $i \in N$ and $P_{i}^{\prime} \in \mathcal{P}$ such that $g\left(P_{i}^{\prime}, P_{-i}\right) P_{i} g\left(P_{i}, P_{-i}\right)$, then there exist $j \in N$ and $P_{j}^{\prime \prime} \in \mathcal{P}$ such that $f\left(P_{j}^{\prime \prime}, P_{-j}\right) P_{j} f\left(P_{j}, P_{-j}\right)$.

Second, and following their definitions in Section 3, a social choice function $f: \mathcal{P}^{n} \rightarrow X$ is at least as strongly manipulable as social choice function $g: \mathcal{P}^{n} \rightarrow X$ according to Pathak and Sönmez (referred to as at least as strongly PS-manipulable as, and written as $f \succeq^{s P S} g$ ) 
if any profile that is vulnerable under $g$ it is also vulnerable under $f$ but the same agent manipulates both social choice functions at the profile; i.e., ${ }^{5}$

- if there exist $i \in N$ and $P_{i}^{\prime} \in \mathcal{P}$ such that $g\left(P_{i}^{\prime}, P_{-i}\right) P_{i} g\left(P_{i}, P_{-i}\right)$, then there exists $P_{i}^{*} \in \mathcal{P}$ such that $f\left(P_{i}^{*}, P_{-i}\right) P_{i} f\left(P_{i}, P_{-i}\right)$.

Proposition 4 below relates the three comparability notions for tops-only social choice functions that are strategy-proof on a restricted and rich domain of preferences. We say that a domain $\widehat{\mathcal{P}}$ of preferences on $X$ is rich if for for every $x \in X$ there exists $P_{i} \in \widehat{\mathcal{P}}$ such that $t\left(P_{i}\right)=x$.

Proposition 4 Let $f: \mathcal{P}^{n} \rightarrow X$ and $g: \mathcal{P}^{n} \rightarrow X$ be two tops-only social choice functions. Assume $f$ and $g$ are strategy-proof on $\widehat{\mathcal{P}} \subsetneq \mathcal{P}$, where $\widehat{\mathcal{P}}$ is rich. Then,

$$
f \succeq^{s P S} g \Rightarrow f \succeq^{P S} g \Rightarrow f \succeq g .
$$

Proof The implication $f \succeq^{s P S} g \Rightarrow f \succeq^{P S} g$ follows immediately from the two definitions. To show that the second implication holds as well, assume $f \succeq^{P S} g$. Fix $i \in N$ and let $P_{i} \in \mathcal{M}_{i}^{g}$. This means that there exists $\left(P_{i}^{\prime}, P_{-i}\right) \in \mathcal{P}^{n}$ such that $g\left(P_{i}^{\prime}, P_{-i}\right) P_{i} g\left(P_{i}, P_{-i}\right)$. Since $g$ is tops-only and $\widehat{\mathcal{P}}$ is rich, we may assume that $P_{-i} \in \widehat{\mathcal{P}}^{n-1}$. By assumption, there exist $j \in N$ and $P_{j}^{\prime \prime} \in \mathcal{P}$ such that

$$
f\left(P_{j}^{\prime \prime}, P_{-j}\right) P_{j} f\left(P_{j}, P_{-j}\right) .
$$

Assume $j \neq i$ and consider any $\widehat{P}_{i} \in \widehat{\mathcal{P}}$ such that $t\left(\widehat{P}_{i}\right)=t\left(P_{i}\right)$; since $\widehat{\mathcal{P}}$ is rich, at least one such preference exists. Set $\widehat{P}=\left(\widehat{P}_{i}, P_{-i}\right) \in \widehat{\mathcal{P}}^{n}$. Since $f$ is tops-only, $f(\widehat{P})=f\left(P_{j}, P_{-j}\right)$. By (29), $j$ can manipulate $f$ at a profile $\widehat{P} \in \widehat{\mathcal{P}}^{n}$, a contradiction with strategy-proofness of $f$ on $\widehat{\mathcal{P}} \subsetneq \mathcal{P}$. Hence, $j=i$. But then, by (29), $P_{i} \in \mathcal{M}_{i}^{f}$. Thus, $P_{i} \in \mathcal{M}_{i}^{f}$ whenever $P_{i} \in \mathcal{M}_{i}^{g}$ which implies that $f$ is at least as manipulable as $g$.

Example 4 below shows that the reverse of the second implication does not hold in our setting where $X=2^{K}$ and the social choice functions under consideration are voting by committees, strategy-proof on the restricted and rich domain of separable preferences. Namely, there exist two voting by committees $\mathcal{W}$ and $\mathcal{V}$ such that $\mathcal{W}$ is at least as manipulable as $\mathcal{V}$ but $\mathcal{W}$ is not at least as PS-manipulable as $\mathcal{V}$ (and, by Proposition 4 above, $\mathcal{W}$ is not at least as strongly PS-manipulable as $\mathcal{V}$ ). In particular, Example 4 together with Proposition 4 show that our notion of being at least as manipulable as is different and strictly stronger than the two notions proposed by Pathak and Sönmez (2013).

\footnotetext{
${ }^{5}$ Observe that the notions of at least as PS-manipulable as and at least as strongly PS-manipulable as are relative to the inclusion of the sets of vulnerable profiles of preferences, while our notion of at least as manipulable as is relative to the inclusion of the sets of manipulable individual preferences.
} 
Example 4 Let $N=\{1,2,3\}$ be the set of agents and $K=\{x, y, z\}$ the set of objects. Consider the two voting by quota $\mathcal{V}$ and $\mathcal{W}$ defined by the following committees:

$$
\begin{aligned}
& \mathcal{V}_{x}^{m}=\mathcal{V}_{y}^{m}=\mathcal{V}_{z}^{m}=\{S \subset N:|S|=2\} \\
& \mathcal{W}_{x}^{m}=\mathcal{W}_{y}^{m}=\mathcal{W}_{z}^{m}=\{S \subset N:|S|=1\}
\end{aligned}
$$

By Corollary $1, \mathcal{V} \succ \mathcal{W}$. On the one hand, consider any profile $P=\left(P_{1}, P_{2}, P_{3}\right) \in \mathcal{P}^{3}$ and any preference $P_{3}^{\prime} \in \mathcal{P}$ such that (i) $t\left(P_{i}\right)=\{z\}$ for $i=1,2$, (ii) $t\left(P_{3}\right)=\{x\}$ and $\{x, y, z\} P_{3}\{x, z\}$, and (iii) $t\left(P_{3}^{\prime}\right)=\{x, y\}$. Therefore, $\mathcal{W}\left(P_{1}, P_{2}, P_{3}^{\prime}\right)=\{x, y, z\} P_{3}\{x, z\}=$ $\mathcal{W}(P)$ and hence, $P$ is vulnerable under $\mathcal{W}$. Moreover, $\mathcal{V}(P)=\{z\}$ and $P$ is not vulnerable under $\mathcal{V}$. Thus, $\mathcal{V}$ is not at least as PS-manipulable as $\mathcal{W}$ and hence, by Proposition $4, \mathcal{V}$ is not at least as strongly PS-manipulable as $\mathcal{W}$. On the other hand, consider any profile $\widehat{P}=$ $\left(\widehat{P}_{1}, \widehat{P}_{2}, \widehat{P}_{3}\right) \in \mathcal{P}^{3}$ and any preference $\widehat{P}_{3}^{\prime} \in \mathcal{P}$ such that (i) $t\left(\widehat{P}_{1}\right)=\{x, z\},\{x, y, z\} \widehat{P}_{1}\{y, z\}$, (ii) $t\left(\widehat{P}_{2}\right)=\{z\}$, (iii) $t\left(\widehat{P}_{3}\right)=\{y\}$ and $\{x, y, z\} \widehat{P}_{3}\{x, z\} \widehat{P}_{3}\{z\}$, and (iv) $t\left(\widehat{P}_{3}^{\prime}\right)=\{x, z\}$. Therefore, $\mathcal{W}(\widehat{P})=\{x, y, z\}$ and $\widehat{P}$ is not vulnerable under $\mathcal{W}$. Moreover, $\mathcal{V}\left(\widehat{P}_{1}, \widehat{P}_{2}, \widehat{P}_{3}^{\prime}\right)=$ $\{x, z\} \widehat{P}_{3}\{z\}=\mathcal{V}(\widehat{P})$ and hence, $\widehat{P}$ is vulnerable under $\mathcal{V}$. Thus, $\mathcal{W}$ is not at least as PSmanipulable as $\mathcal{V}$; hence, by Proposition $4, \mathcal{W}$ is not at least as strongly PS-manipulable as $\mathcal{V}$. Therefore, $\mathcal{W}$ and $\mathcal{V}$ are not comparable according to the two notions proposed by Pathak and Sönmez (2013).

\section{References}

[1] R. Pablo Arribillaga and Jordi Massó. 2016. "Comparing Generalized Median Voter Schemes According to Their Manipulability." Theoretical Economics 11: 547586 .

[2] Salvador Barberà, Jordi Massó, and Alejandro Neme. 1999. "Maximal Domains of Preferences Preserving Strategy-proofness for Generalized Median Voter Schemes." Social Choice and Welfare 16 (2): 321-336.

[3] Salvador Barberà, Hugo F. Sonnenschein, and Lin Zhou. 1991. "Voting by Committees." Econometrica 59 (3): 595-609.

[4] Donald E. Campbell and Jerry S. Kelly. 2009. "Gains from Manipulating Social Choice Rules." Economic Theory 40 (3): 349-371.

[5] Gabriel Carroll. 2013. "A Quantitative Approach to Incentives: Application to Voting Rules." Mimeo. 
[6] Peter Chen, Michael Egesdal, Marek Pycia, and M. Bumin Yenmez. 2016. "Manipulability of Stable Mechanisms." American Economic Journal: Microeconomics 8 (2): 202-214.

[7] Lars Ehlers, Hans Peters, and Ton Storcken. 2004. "Threshold Strategy-proofness: on Manipulability in Large Voting Problems." Games and Economic Behavior 49 (1): $103-116$.

[8] Jerry S. Kelly. 1993. "Almost all Social Choice Rules Are Highly Manipulable, But a Few Aren't," Social Choice and Welfare 10 (2): 161-175.

[9] Stefan Maus, Hans Peters, and Ton Storcken. 2007a. "Anonymous Voting and Minimal Manipulability." Journal of Economic Theory 135 (1): 533-544.

[10] Stefan Maus, Hans Peters, and Ton Storcken. 2007b. "Minimal Manipulability: Unanimity and Nondictatorship." Journal of Mathematical Economics 43 (6): 675-691.

[11] Stefan Maus, Hans Peters, and Ton Storcken. 2007c. "Minimally Manipulable Anonymous Social Choice Functions." Mathematical Social Sciences 53 (3): 239-254.

[12] Stefan Maus, Hans Peters, and Ton Storcken. 2007d. "Minimal Manipulability: Anonymity and Unanimity." Social Choice and Welfare 29 (2): 247-269.

[13] Shmuel Nitzan. 1985. "The Vulnerability of Point-voting Schemes to Preference Variation and Strategic Manipulation." Public Choice 47 (2): 349-370.

[14] Parag Pathak and Tayfun Sönmez. 2013. "School Admissions Reform in Chicago and England: Comparing Mechanisms by Their Vulnerability to Manipulation." American Economic Review 103 (1): 80-106.

[15] Bezalel Peleg. 1979. "A Note on Manipulability of Large Voting Schemes." Theory and Decision 11 (4): 401-412.

[16] Shigehiro Serizawa. 1995. "Power of Voters and Domain of Preferences where Voting by Committees Is Strategy-proof." Journal of Economic Theory 67 (2): 599-608.

[17] David A. Smith. 1999. "Manipulability Measures of Common Social Choice Functions." Social Choice and Welfare 16 (4): 639-661. 